\title{
Fractional order ecological system for complexities of interacting species with harvesting threshold in imprecise environment
}

\author{
Najeeb Alam Khan ${ }^{1 *}$ (D), Oyoon Abdul Razzaq ${ }^{2}$, Sankar Parsad Mondal ${ }^{3}$ and Qammar Rubbab ${ }^{4}$
}

\section{"Correspondence: \\ njbalam@yahoo.com \\ 'Department of Mathematics, \\ University of Karachi, Karachi, \\ Pakistan \\ Full list of author information is \\ available at the end of the article}

\section{Springer}

\begin{abstract}
The key objective of this paper is to study the imprecise biological complexities in the interaction of two species pertaining to harvesting threshold. It is explained by taking the prey-predator model with imprecise biological parameters and fractional order generalized Hukuhara $(\mathrm{fgH})$ differentiability. In this vain, different possible systems of the model are constructed, according to the increasing and decreasing behavior of population growth. Feasibility and stability analyses of equilibrium points of the stated models are also discussed by means of variational matrix with Routh-Hurwitz conditions. In addition, the numerical elaborations are carried out by taking parametric expansion of fuzzy fractional Laplace transform (FFLT). This significantly helps the researchers in using a novel approach to analyze the constant solutions of the dynamical systems in the presence of fractional index. This would allow the avoidance of any intricacy that occurs while solving fractional order derivatives. Furthermore, this attempt also provides numerical and pictorial results, obtained through some well-known methods, namely fifth-forth Runge-Kutta method (FFRK), Grunwald-Letnikov's definition (GL) and Adams-Bashforth method (ABM) that are deemed appropriate to scrutinize the dynamics of the system of equations.
\end{abstract}

Keywords: Fractional generalized Hukuhara differentiable; Routh-Hurwitz condition; Triangular fuzzy number; Fuzzy fractional Laplace transform; Stability analysis

\section{Introduction}

There has been considerable work done by researchers in associating real ecological situations with mathematical models to make it palpable. The model for population growth of biological species, named the Lotka-Volterra equation, played an important role in mathematical biology [1, 2]. These equations, with interspecific competition, have been considered to be a prerequisite for those which are associated with biology. These equations also aid the perceptions about the outcomes of competitive interactions between different species [3-6]. Diversity of factors, such as environmental change, consumer-resource interactions, and disease, not covered in the model can affect the upshot of competitive interactions by affecting the dynamics of the respective populations. In theoretical ecology,

(c) The Author(s) 2019. This article is distributed under the terms of the Creative Commons Attribution 4.0 International License (http://creativecommons.org/licenses/by/4.0/), which permits unrestricted use, distribution, and reproduction in any medium, provided you give appropriate credit to the original author(s) and the source, provide a link to the Creative Commons license, and indicate if changes were made. 
many researchers have exhibited their models with exactly known biological parameters: however, in reality, the values of all parameters cannot be known accurately for various reasons like the lack of data, scarcity of information, oversight in the measurement operation and determining the initial conditions, etc. In order to overcome these limitations, these models are deliberated with imprecise parameters to produce more realistic and favorable results. However, there are numerous ways to deal with models with imprecision in parameters. Fuzzy theory is introduced to be utilized to a great extent. In this theory, the parameters are replaced by fuzzy numbers or fuzzy functions that handle the imprecision. Despite of being very challenging, numerous remarkable innovations have caught the sight of many researchers. For instance, Pal et al. [7] proposed three species preypredator harvesting model and used the logical hybridizing of the fuzzy numbers to derive optimal equilibrium points and harvesting efforts. Nounou et al. [8] illustrated glycolyticglycogenolytic pathway model, purine metabolism pathway model, and a genetic pathway using fuzzy systems strategy and developed model-free nonlinear intervention strategies. Paul et al. [9] discussed the fuzzy quota harvesting of a single species Lotka-Volterra equation using generalized Hukuhara differentiability. Thus, many authors have utilized this concept for modeling in mathematical biology [10-14].

Since the last decade, the phenomena of fractional calculus in modeling different aspects of real world have been widely increasing. For its nonlocal properties, this theory has provided an exceptional tool in the interest of the scientific community. Khan et al. [15] discussed a system of nonlinear fractional differential equations with imprecision and performed Grunwald-Letnikov's definition for the analysis. Kumar et al. [16] did an empirical study for hyperthermia treatment therapy by using time fractional dual-phase-lag bioheat transfer model. Meng et al. [17] described the efficiency of the variable order fractional calculus for the prediction of the compression deformation of amorphous glassy polymers. Singh [18] studied the dynamics of the rumor spreading in a social network by means of Atangana-Baleanu derivative of non-integer order. He analyzed the effect of the fractional order on various human behaviors such as ignorant, spreading, and stifles. Thus, applications of heterogeneous fractional based equations, for instance, linear and nonlinear fractional ordinary and partial differential equations, fuzzy fractional differential equations, a system of fractional equations etc., greatly exist in various scientific disciplines [19-31].

Although some attempts have already been made [7, 10-14], in this endeavor we carried out the investigations with some new inputs and concepts, which brings a novelty in this framework and provides appropriate applications. The key elements are described as follows:

- The model is structured with the concept of fgH-differentiability.

- Elaborate conditions for the harvesting threshold affecting the population dynamics of both species are given.

- The pioneering numerical illustrations of equilibrium points in the presence of fractional index are incorporated.

- Innovatively, parametric expansion of fuzzy fractional Laplace transform is explained to deal with the fractional order derivatives.

- It also contains numerical study of different techniques for solving fuzzy fractional dynamical models. 
The model is classified with increasing and decreasing behavior of population growth of the species, which may transpire due to the climate change, with the help of fgHdifferentiability [31-33]. This notion clearly describes all the possible cases of differentiability of the fuzzy functions by breaking it into multiple systems of equations, where each system elaborates a different scenario. These governing systems, which are advantageous to be associated with the dynamics of any species, are taken into account. It also has the capability to measure the linguistic phrases that are widely used for explaining any imprecise attribute, e.g., a minor growth, very strong carrying capacity, etc.: whereas the fractional derivative is substantially beneficial to scrutinizing the gradual rate of change in per capita growth by detecting each slight change of the dynamical system. Moreover, the significance of our model on including fractional and fuzzy perceptions with harvesting threshold can be easily understood by considering a very common paradigm of ecological study, i.e., the dynamics of caribou and wolf. If we take the example of Arctic people, they are dependent on caribou for food, clothing, and shelter, so the hunting of caribou takes place widely in this region. Since caribou's body composition varies highly with season, uncertainty occurs in their intrinsic growth or carrying capacity as the weather goes through uncertain variations. Analogously, due to many reasons such as overhunting by humans, catching bloodsucking diseases, or mating season, their population density possesses either increasing or decreasing patterns. On the other hand, the wolves, which are hunted by humans to save their livestock or for draught or security purposes in army in some regions, are considered as predator. Their population density also varies due to nutritional stress, diseases, and parasites or any other environmental change. It is widely known that wolves become more active in autumn-spring season, therefore their intrinsic body also alters within a year. So, it is more suitable to define the parameters with uncertain values, which explains their level of increase or decrease, accordingly. As a consequence of the aforementioned uncertainties, a minor change within a system gradually leads to major change that may cause positive or negative impact on a body. Thus, this fractional rate of change further remarkably discusses these minor changes along with the fuzziness occurring within the body, instead of directly calculating a whole change. Moreover, to cope with the fractional operator, we implement the parametric expansion of fuzzy fractional Laplace transform $[31,34,35]$. The expansion of fractional Laplace transform greatly converts the fractional order derivative into the integer order, which can be further scrutinized easily using any appropriate numerical-analytical methods. By means of variational matrices in conjunction with Routh-Hurwitz conditions [36, 37], the analysis of stability of equilibrium solutions is illustrated. Some equilibrium points with fractional index are also obtained to further study the historical state of constant solutions. Additionally, we explore a comparative analysis between FFRK, Grunwald-Letnikov's definition (GL) [15], and Adams-Bashforth method (ABM) [38]. These techniques are widely known approximators to fractional and integer order differential equations. These assessments are examined on some illustrative examples and lucratively depicted increase or decrease in the populations, phase trajectories, and limit cycles etc., of the systems.

Furthermore, the remaining structure of the paper is organized as follows. Detailed discussion on modeling of the prey-predator model using fgH-differentiability is described in Sect. 2 and existence of equilibrium points is elaborated in Sect. 3. The stability analysis for each equilibrium point along with the conditions for the harvesting parameter is given in Sect. 4. Section 5 contains the implementation of FFLT, some numerical examples, and 
graphical deliberations, using FFRK, GL, and ABM methods. Finally, Sect. 6 concludes the major findings of this work.

\section{Prey-predator model formulation}

Consider the following prey-predator model:

$$
\begin{aligned}
& { }^{C} D_{t}^{\sigma} x(t)=x(t)\left(r_{1}-\frac{b_{11}}{k_{1}} x(t)-b_{12} y(t)-q_{1} E\right), \\
& { }^{C} D_{t}^{\sigma} y(t)=y(t)\left(r_{2}-\frac{b_{22}}{k_{2}} y(t)+b_{21} x(t)-q_{2} E\right),
\end{aligned}
$$

where ${ }^{C} D_{t}^{\sigma}$ represents Caputo-type fractional-order derivative for $0<\sigma \leq 1, x(t)$ and $y(t)$ represent the population density of prey and predator with the fractional variation in time, respectively. In addition, $r_{i}, k_{i}, b_{i j}$, and $q_{i}$ for $i, j=1,2$ are all positive constants such that $r_{i}$ are the intrinsic growth rates, $k_{i}$ are the carrying capacities, $b_{i j}$ for $i=j$ are the coefficients of interspecific competition, $b_{i j}$ for $i \neq j$ are the extents to which the availability of $j$ th species affects the growth of $i$ th species, $q_{i}$ are the catchability coefficients of both species. Furthermore, with the assumption that prey and predator are highly demanding species, $E$ is considered as the harvesting threshold of individuals over time for both the species. More often, environmental or climate changes may induce imprecise upshots in the population dynamics of the species. Therefore, taking the population of both species in a crisp sense is not of interest nowadays. For instance, the carrying capacity $k$ of any species defines the maximum population of that species sustained by the environment. After a time lag, it may slightly overshoot or undershoot due to the fluctuations in environmental resources, but does not remain constant. Similarly, with the other parameters, they are greatly affected by the encircling ecology, which itself is an imprecise context. Hence, restructuring system (1), by considering $x(t)$ and $y(t)$ to be non-negative fuzzy functions and all the parameters as fuzzy numbers except $q_{1}, q_{2}$, and $E$, the above system turns into

$$
\begin{aligned}
& { }^{C} D_{t}^{\sigma} \tilde{x}(t)=\tilde{x}(t)\left(\tilde{r}_{1}-\frac{\tilde{b}_{11}}{\tilde{k}_{1}} \tilde{x}(t)-\tilde{b}_{12} \tilde{y}(t)-q_{1} E\right), \\
& { }^{C} D_{t}^{\sigma} \tilde{y}(t)=\tilde{y}(t)\left(\tilde{r}_{2}-\frac{\tilde{b}_{22}}{\tilde{k}_{2}} \tilde{y}(t)+\tilde{b}_{21} \tilde{x}(t)-q_{2} E\right),
\end{aligned}
$$

with the initial conditions $\tilde{x}\left(t_{0}\right)=\tilde{x}_{0}$ and $\tilde{y}\left(t_{0}\right)=\tilde{y}_{0}$. From the properties of fuzzy calculus [31, 33], fuzzy functions and parameters can be represented in $\alpha$-levels as $[\tilde{x}(t)]^{\alpha}=$ $\left[x_{l}(t ; \alpha), x_{u}(t ; \alpha)\right],[\tilde{y}(t)]^{\alpha}=\left[y_{l}(t ; \alpha), y_{u}(t ; \alpha)\right],\left[\tilde{r}_{i}\right]^{\alpha}=\left[r_{i l}(\alpha), r_{i u}(\alpha)\right],\left[\tilde{b}_{i j}\right]^{\alpha}=\left[b_{i j l}(\alpha), b_{i j u}(\alpha)\right]$, $\left[\tilde{k}_{i}\right]^{\alpha}=\left[k_{i l}(\alpha), k_{i u}(\alpha)\right]$, with fuzzy initial conditions $\left[\tilde{x}_{0}\right]^{\alpha}=\left[x_{0 l}(\alpha), x_{0 u}(\alpha)\right]$ and $\left[\tilde{y}_{0}\right]^{\alpha}=$ $\left[y_{0 l}(\alpha), y_{0 u}(\alpha)\right]$, for all $\alpha \in[0,1]$ and $i, j=1,2$. It is well known that $\left(\Lambda_{F}, \mathbf{D}\right)$ postulates a complete metric space, where $\Lambda_{F}$ is the fuzzy space and $\mathbf{D}$ symbolizes the Hausdorff metric [15]. Since the right-hand side of Eq. (2) is continuous, system (2) satisfies the following theorem of Lipschitz condition, which concludes the stability and uniqueness of the system. 
Theorem 2.1 Let $\tilde{f}$ be a fuzzy function defined as $\tilde{f}: \Re \rightarrow \Lambda_{F}$, then $\tilde{f}$ is Lipschitz continuous if there exists a real constant $L>0$ such that, for all $\tilde{x}$ and $\tilde{y}$ in $\Lambda_{F}$,

$$
\mathbf{D}(\tilde{f}(\tilde{x}), \tilde{f}(\tilde{y})) \leq L \mathbf{D}(\tilde{x}, \tilde{y})
$$

The detailed proof of the theorem is found in many research papers [39, 40].

Moreover, using the concept of fgH-differentiability [31-33] and considering all the possible cases of fgH-differentiability of $\tilde{x}(t)$ and $\tilde{y}(t)$, fuzzy model (2) can be further expanded as, for all $\alpha \in[0,1]$,

(a) If both $\tilde{x}(t)$ and $\tilde{y}(t)$ are (i)-fgH-differentiable, then

$$
\begin{aligned}
& { }^{C} D_{t}^{\sigma} x_{l}(t ; \alpha)=x_{l}(t ; \alpha)\left(r_{1 l}(\alpha)-\frac{b_{11 u}(\alpha)}{k_{1 u}(\alpha)} x_{u}(t ; \alpha)-b_{12 u}(\alpha) y_{u}(t ; \alpha)-q_{1} E\right), \\
& { }^{C} D_{t}^{\sigma} x_{u}(t ; \alpha)=x_{u}(t ; \alpha)\left(r_{1 u}(\alpha)-\frac{b_{11 l}(\alpha)}{k_{1 l}(\alpha)} x_{l}(t ; \alpha)-b_{12 l}(\alpha) y_{l}(t ; \alpha)-q_{1} E\right), \\
& { }^{C} D_{t}^{\sigma} y_{l}(t ; \alpha)=y_{l}(t ; \alpha)\left(r_{2 l}(\alpha)-\frac{b_{22 u}(\alpha)}{k_{2 u}(\alpha)} y_{u}(t ; \alpha)+b_{21 l}(\alpha) x_{l}(t ; \alpha)-q_{2} E\right), \\
& { }^{C} D_{t}^{\sigma} y_{u}(t ; \alpha)=y_{u}(t ; \alpha)\left(r_{2 u}(\alpha)-\frac{b_{22 l}(\alpha)}{k_{2 l}(\alpha)} y_{l}(t ; \alpha)+b_{21 u}(\alpha) x_{u}(t ; \alpha)-q_{2} E\right) .
\end{aligned}
$$

(b) If $\tilde{x}(t)$ is (i)-fgH-differentiable and $\tilde{y}(t)$ is (ii)-fgH-differentiable, then

$$
\begin{aligned}
& { }^{C} D_{t}^{\sigma} x_{l}(t ; \alpha)=x_{l}(t ; \alpha)\left(r_{1 l}(\alpha)-\frac{b_{11 u}(\alpha)}{k_{1 u}(\alpha)} x_{u}(t ; \alpha)-b_{12 u}(\alpha) y_{l}(t ; \alpha)-q_{1} E\right), \\
& { }^{C} D_{t}^{\sigma} x_{u}(t ; \alpha)=x_{u}(t ; \alpha)\left(r_{1 u}(\alpha)-\frac{b_{11 l}(\alpha)}{k_{1 l}(\alpha)} x_{l}(t ; \alpha)-b_{12 l}(\alpha) y_{u}(t ; \alpha)-q_{1} E\right), \\
& { }^{C} D_{t}^{\sigma} y_{l}(t ; \alpha)=y_{u}(t ; \alpha)\left(r_{2 u}(\alpha)-\frac{b_{22 l}(\alpha)}{k_{2 l}(\alpha)} y_{l}(t ; \alpha)+b_{21 l}(\alpha) x_{l}(t ; \alpha)-q_{2} E\right), \\
& { }^{C} D_{t}^{\sigma} y_{u}(t ; \alpha)=y_{l}(t ; \alpha)\left(r_{2 l}(\alpha)-\frac{b_{22 u}(\alpha)}{k_{2 u}(\alpha)} y_{u}(t ; \alpha)+b_{21 u}(\alpha) x_{u}(t ; \alpha)-q_{2} E\right) .
\end{aligned}
$$

(c) If $\tilde{x}(t)$ is (ii)-fgH-differentiable and $\tilde{y}(t)$ is (i)-fgH-differentiable, then

$$
\begin{aligned}
& { }^{C} D_{t}^{\sigma} x_{l}(t ; \alpha)=x_{u}(t ; \alpha)\left(r_{1 u}(\alpha)-\frac{b_{11 l}(\alpha)}{k_{1 l}(\alpha)} x_{l}(t ; \alpha)-b_{12 l}(\alpha) y_{l}(t ; \alpha)-q_{1} E\right), \\
& { }^{C} D_{t}^{\sigma} x_{u}(t ; \alpha)=x_{l}(t ; \alpha)\left(r_{1 l}(\alpha)-\frac{b_{11 u}(\alpha)}{k_{1 u}(\alpha)} x_{u}(t ; \alpha)-b_{12 u}(\alpha) y_{u}(t ; \alpha)-q_{1} E\right), \\
& { }^{C} D_{t}^{\sigma} y_{l}(t ; \alpha)=y_{l}(t ; \alpha)\left(r_{2 l}(\alpha)-\frac{b_{22 u}(\alpha)}{k_{2 u}(\alpha)} y_{u}(t ; \alpha)+b_{21 u}(\alpha) x_{u}(t ; \alpha)-q_{2} E\right), \\
& { }^{C} D_{t}^{\sigma} y_{u}(t ; \alpha)=y_{u}(t ; \alpha)\left(r_{2 u}(\alpha)-\frac{b_{22 l}(\alpha)}{k_{2 l}(\alpha)} y_{l}(t ; \alpha)+b_{21 l}(\alpha) x_{l}(t ; \alpha)-q_{2} E\right) .
\end{aligned}
$$


(d) If both $\tilde{x}(t)$ and $\tilde{y}(t)$ are (ii)-fgH-differentiable, then

$$
\begin{aligned}
& { }^{C} D_{t}^{\sigma} x_{l}(t ; \alpha)=x_{u}(t ; \alpha)\left(r_{1 u}(\alpha)-\frac{b_{11 l}(\alpha)}{k_{1 l}(\alpha)} x_{l}(t ; \alpha)-b_{12 l}(\alpha) y_{l}(t ; \alpha)-q_{1} E\right), \\
& { }^{C} D_{t}^{\sigma} x_{u}(t ; \alpha)=x_{l}(t ; \alpha)\left(r_{1 l}(\alpha)-\frac{b_{11 l}(\alpha)}{k_{1 l}(\alpha)} x_{l}(t ; \alpha)-b_{12 l}(\alpha) y_{l}(t ; \alpha)-q_{1} E\right), \\
& { }^{C} D_{t}^{\sigma} y_{l}(t ; \alpha)=y_{u}(t ; \alpha)\left(r_{2 u}(\alpha)-\frac{b_{22 l}(\alpha)}{k_{2 l}(\alpha)} y_{l}(t ; \alpha)+b_{21 l}(\alpha) x_{l}(t ; \alpha)-q_{2} E\right), \\
& { }^{C} D_{t}^{\sigma} y_{u}(t ; \alpha)=y_{l}(t ; \alpha)\left(r_{2 l}(\alpha)-\frac{b_{22 u}(\alpha)}{k_{2 u}(\alpha)} y_{u}(t ; \alpha)+b_{21 u}(\alpha) x_{u}(t ; \alpha)-q_{2} E\right),
\end{aligned}
$$

with the same initial conditions as outlined for system (2).

\section{Equilibrium points}

In this section, equilibrium points of each fgH-differential system (3)-(6) are illustrated in conjunction with the feasibility conditions. In view of the fact that equilibrium points represent the constant solutions of the system, assume ${ }^{C} D^{\sigma} x_{l}(t ; \alpha)=0,{ }^{C} D^{\sigma} x_{u}(t ; \alpha)=0$, ${ }^{C} D^{\sigma} y_{l}(t ; \alpha)=0$, and ${ }^{C} D^{\sigma} y_{u}(t ; \alpha)=0$ in systems (3)-(6). On solving the remaining nonlinear equations on the right-hand side, the following equilibrium points for positive functions are attained.

\subsection{Trivial equilibrium point}

The trivial equilibrium points, $(0,0,0,0)$ are always feasible for each system (3)-(6). Let, these points be symbolized as, $e_{10}\left(x_{10 l}, x_{10 u}, y_{10 l}, y_{10 u}\right), e_{20}\left(x_{20 l}, x_{20 u}, y_{20 l}, y_{20 u}\right), e_{30}\left(x_{30 l}, x_{30 u}\right.$, $\left.y_{30 l}, y_{30 u}\right)$ and $e_{40}\left(x_{40 l}, x_{40 u}, y_{40 l}, y_{40 u}\right)$ for systems (3)-(6), respectively.

\subsection{Axial equilibrium point}

Let the axial equilibria be denoted by $e_{11}\left(x_{11 l}, x_{11 u}, y_{11 l}, y_{11 u}\right), e_{21}\left(x_{21 l}, x_{21 u}, y_{21 l}, y_{21 u}\right)$, $e_{31}\left(x_{31 l}, x_{31 u}, y_{31 l}, y_{31 u}\right)$, and $e_{41}\left(x_{41 l}, x_{41 u}, y_{41 l}, y_{41 u}\right)$ for systems (3)-(6), respectively. For the positive functions, each system (3)-(6) produces axial equilibrium point $\left(\frac{k_{1 l}\left(r_{1 u}-E q_{1}\right)}{b_{11 l}}\right.$, $\left.\frac{k_{1 u}\left(r_{1 l}-E q_{1}\right)}{b_{11 u}}, 0,0\right)$. This point is feasible if the harvesting threshold satisfies the following conditions:

$$
E<\frac{r_{1 u}}{q_{1}} \text { and } E<\frac{r_{1 l}}{q_{1}}, \quad \text { i.e. } E<\min \left(\frac{r_{1 l}}{q_{1}}, \frac{r_{1 u}}{q_{1}}\right)
$$

\subsection{Coexistence equilibrium point}

Assume that $e_{12}\left(x_{12 l}, x_{12 u}, y_{12 l}, y_{12 u}\right), e_{22}\left(x_{22 l}, x_{22 u}, y_{22 l}, y_{22 u}\right), e_{32}\left(x_{32 l}, x_{32 u}, y_{32 l}, y_{32 u}\right)$, and $e_{42}\left(x_{42 l}, x_{42 u}, y_{42 l}, y_{42 u}\right)$ represent the coexistence equilibria of systems (3)-(6), respectively, then

$$
\begin{aligned}
& x_{12 l}=\frac{k_{1 l}\left(b_{22 l} r_{1 u}-b_{12 l} k_{2 l} r_{2 u}-E b_{22 l} q_{1}+E b_{12 l} k_{2 l} q_{2}\right)}{b_{11 l} b_{22 l}+b_{12 l} b_{21 l} k_{1 l} k_{2 l}}, \\
& x_{12 u}=\frac{k_{1 u}\left(b_{22 u} r_{1 l}-b_{12 u} k_{2 u} r_{2 l}-E b_{22 u} q_{1}+E b_{12 u} k_{2 u} q_{2}\right)}{b_{11 u} b_{22 u}+b_{12 u} b_{21 u} k_{1 u} k_{2 u}}, \\
& y_{12 l}=\frac{k_{2 l}\left(b_{11 l} r_{2 u}+b_{21 l} k_{1 l} r_{1 u}-E b_{11 l} q_{2}-E b_{21 l} k_{1 l} q_{1}\right)}{b_{11 l} b_{22 l}+b_{12 l} b_{21 l} k_{2 l} k_{1 l}},
\end{aligned}
$$


and

$$
y_{12 u}=\frac{k_{2 u}\left(b_{11 u} r_{2 l}+b_{21 u} k_{1 u} r_{1 l}-E b_{11 u} q_{2}-E b_{21 u} k_{1 u} q_{1}\right)}{b_{11 u} b_{22 u}+b_{12 u} b_{21 u} k_{2 u} k_{1 u}}
$$

are coexistence equilibrium points of system (3), and it is feasible if

$$
\begin{aligned}
& \max \left(\frac{b_{12 l} k_{2 l} r_{2 u}-b_{22 l} r_{1 u}}{b_{12 l} k_{2 l} q_{2}-b_{22 l} q_{1}}, \frac{b_{12 u} k_{2 u} r_{2 l}-b_{22 u} r_{1 l}}{b_{12 u} k_{2 u} q_{2}-b_{22 u} q_{1}}\right) \\
& <E<\min \left(\frac{b_{12 l} k_{2 l} r_{2 u}-b_{22 l} r_{1 u}}{b_{12 l} k_{2 l} q_{2}-b_{22 l} q_{1}}, \frac{b_{12 u} k_{2 u} r_{2 l}-b_{22 u} r_{1 l}}{b_{12 u} k_{2 u} q_{2}-b_{22 u} q_{1}}\right) ; \quad \text { whereas } \\
& x_{22 l}=\frac{k_{1 l}\left(b_{22 l} r_{1 u}-b_{12 l} k_{2 l} r_{2 u}-E b_{22 u} q_{1}+E b_{12 l} k_{2 l} q_{2}\right)}{b_{11 l} b_{22 u}+b_{12 l} b_{21 l} k_{1 l} k_{2 l}}, \\
& x_{22 u}=\frac{k_{1 u}\left(b_{22 u} r_{1 l}-b_{12 u} k_{2 u} r_{2 l}-E b_{22 u} q_{1}+E b_{12 u} k_{2 u} q_{2}\right)}{b_{11 u} b_{22 u}+b_{12 u} b_{21 u} k_{1 u} k_{2 u}} \\
& y_{22 l}=\frac{k_{2 l}\left(b_{11 l} r_{2 u}+b_{21 l} k_{1 l} r_{1 u}-E b_{11 l} q_{2}-E b_{21 l} k_{1 l} q_{1}\right)}{b_{11 l} b_{22 u}+b_{12 l} b_{21 l} k_{2 l} k_{1 l}}
\end{aligned}
$$

and

$$
y_{22 u}=\frac{k_{2 u}\left(b_{11 u} r_{2 l}+b_{21 u} k_{1 u} r_{1 l}-E b_{11 u} q_{2}-E b_{21 u} k_{1 u} q_{1}\right)}{b_{11 u} b_{22 u}+b_{12 u} b_{21 u} k_{2 u} k_{1 u}}
$$

are coexistence equilibrium points of system (4), and it is feasible if

$$
\begin{aligned}
& \max \left(\frac{b_{12 l} k_{2 l} r_{2 u}-b_{22 l} r_{1 u}}{b_{12 l} k_{2 l} q_{2}-b_{22 u} q_{1}}, \frac{b_{12 u} k_{2 u} r_{2 l}-b_{22 u} r_{1 l}}{b_{12 u} k_{2 u} q_{1}-b_{11 u} q_{2}}\right) \\
& <E<\min \left(\frac{b_{21 l} k_{1 l} r_{1 u}+b_{11 l} r_{2 u}}{b_{21 l} k_{1 l} q_{1}+b_{11 l} q_{2}}, \frac{b_{21 u} k_{1 u} r_{1 l}+b_{11 u} r_{2 l}}{b_{21 u} k_{1 u} q_{1}+b_{11 u} q_{2}}\right) .
\end{aligned}
$$

Similarly,

$$
\begin{aligned}
& x_{32 l}=\frac{k_{1 l}\left(b_{22 u} r_{1 u}-b_{12 l} k_{2 l} r_{2 u}-E b_{22 l} q_{1}+E b_{12 l} k_{2 l} q_{2}\right)}{b_{11 l} b_{22 u}+b_{12 l} b_{21 l} k_{1 l} k_{2 l}}, \\
& x_{32 u}=\frac{k_{1 u}\left(b_{22 u} r_{1 l}-b_{12 u} k_{2 u} r_{2 l}-E b_{22 u} q_{1}+E b_{12 u} k_{2 u} q_{2}\right)}{b_{11 u} b_{22 u}+b_{12 l} b_{21 l} k_{1 l} k_{2 l}}, \\
& y_{32 l}=\frac{k_{2 l}\left(b_{11 l} r_{2 u}+b_{21 l} k_{1 l} r_{1 u}-E b_{11 l} q_{2}-E b_{21 l} k_{1 l} q_{1}\right)}{b_{11 l} b_{22 u}+b_{12 l} b_{21 l} k_{2 l} k_{1 l}},
\end{aligned}
$$

and

$$
y_{32 u}=\frac{k_{2 u}\left(b_{11 u} r_{2 l}+b_{21 u} k_{1 u} r_{1 l}-E b_{11 u} q_{2}-E b_{21 u} k_{1 u} q_{1}\right)}{b_{11 u} b_{22 u}+b_{12 u} b_{21 u} k_{2 u} k_{1 u}}
$$

are of system (5), and it is feasible if

$$
\begin{aligned}
& \max \left(\frac{b_{12 u} k_{2 u} r_{2 l}-b_{22 u} r_{1 l}}{b_{12 u} k_{2 u} q_{2}-b_{22 u} q_{1}}, \frac{b_{12 l} k_{2 l} r_{2 u}-b_{22 u} r_{1 u}}{b_{12 l} k_{2 l} q_{2}-b_{22 l} q_{1}}\right) \\
& <E<\min \left(\frac{b_{21 l} k_{1 l} r_{1 u}+b_{11 l} r_{2 u}}{b_{21 l} k_{1 l} q_{1}+b_{11 l} q_{2}}, \frac{b_{21 u} k_{1 u} r_{1 l}+b_{11 u} r_{2 l}}{b_{21 u} k_{1 u} q_{1}+b_{11 u} q_{2}}\right) .
\end{aligned}
$$


In addition,

$$
\begin{aligned}
& x_{42 l}=\frac{k_{2 u}\left(b_{11 u} r_{2 l}+b_{21 u} k_{1 u} r_{1 l}-E b_{11 u} q_{2}-E b_{21 u} k_{1 u} q_{1}\right)}{b_{11 u} b_{22 u}+b_{12 u} b_{21 u} k_{1 u} k_{2 u}}, \\
& x_{42 u}=\frac{k_{1 l}\left(b_{11 l} r_{2 u}+b_{21 l} k_{1 l} r_{1 u}-E b_{11 l} q_{2}-E b_{21 l} k_{1 l} q_{1}\right)}{b_{11 l} b_{22 u}+b_{12 l} b_{21 l} k_{1 l} k_{2 l}}, \\
& y_{42 l}=\frac{k_{1 u}\left(b_{22 u} r_{1 l}-b_{12 u} k_{2 u} r_{2 l}-E b_{22 u} k_{1 u} q_{1}+E b_{12 u} k_{1 u} k_{2 u} q_{2}\right)}{b_{11 l} b_{22 u}+b_{12 l} b_{21 l} k_{2 l} k_{1 l}},
\end{aligned}
$$

and

$$
y_{42 u}=\frac{k_{1 l}\left(b_{22 u} r_{1 u}-b_{12 l} k_{2 l} r_{2 u}-E b_{22 u} q_{1}+E b_{12 l} k_{2 l} q_{2}\right)}{b_{11 l} b_{22 u}+b_{12 l} b_{21 l} k_{2 l} k_{1 l}}
$$

are of system (6), and it is feasible if

$$
\begin{aligned}
E> & \max \left(\frac{b_{12 l} k_{1 l} k_{2 l} r_{2 u}-b_{22 l} k_{1 l} r_{1 u}}{b_{12 l} k_{1 l} k_{2 l} q_{2}-b_{22 l} k_{1 l} q_{1}}, \frac{b_{12 u} k_{1 u} k_{2 u} r_{2 l}-b_{22 u} k_{1 u} r_{1 l}}{b_{12 u} k_{1 u} k_{2 u} q_{2}-b_{22 u} k_{1 u} q_{1}},\right. \\
& \left.\frac{b_{21 l} k_{2 l} k_{1 l} r_{1 u}-b_{11 l} k_{2 l} r_{2 u}}{b_{21 l} k_{2 l} k_{1 l} q_{1}-b_{11 l} k_{2 l} q_{2}}, \frac{b_{21 u} k_{2 u} k_{1 u} r_{1 l}-b_{11 u} k_{2 u} r_{2 l}}{b_{21 u} k_{2 u} k_{1 u} q_{1}-b_{11 u} k_{2 u} q_{2}}\right) .
\end{aligned}
$$

\section{Stability analysis}

In this section, further classification of the equilibrium points is done with respect to the local stability by constructing variational matrix at each equilibrium point and then calculating eigenvalues $\lambda$ in conjunction with Routh-Hurwitz conditions [36, 37]. Accordingly, if all the calculated eigenvalues $\lambda_{j},(j=1,2,3,4)$, for an equilibrium point are negative real numbers, then the point is said to be stable or asymptotically stable. On the other hand, if there exists at least one positive eigenvalue, then the equilibrium point is considered to be unstable. Besides, if a pair of purely imaginary eigenvalues is attained with all other negative real numbers, then the system might produce limit cycles. Also, in case of characteristic equation of the variational matrix, Routh-Hurwitz condition states that if the coefficients $\beta_{n},(n=1,2,3,4)$, fulfill the conditions $\beta_{1}>0, \beta_{2}>0, \beta_{3}>0$, and $\beta_{1} \beta_{2} \beta_{3}>\beta_{3}^{2}+\beta_{1}^{2} \beta_{4}$, then the equilibrium point is considered to be stable.

Now, consider all the fgH-differential systems (3)-(6), then the variational matrices are constructed follows:

$$
\begin{aligned}
& \mathbf{V}_{1}=\left(\begin{array}{cccc}
r_{1 l}-\frac{b_{11 u}}{k_{1 u}} x_{u}-b_{12 u} y_{u}-q_{1} E & -\frac{b_{11 u}}{k_{1 u}} x_{l} & 0 & -b_{12 u} x_{l} \\
-\frac{b_{111}}{k_{1 l} l} x_{u} & r_{1 u}-\frac{b_{111}}{k_{1 l}} x_{l}-b_{12 l} y_{l}-q_{1} E & -b_{12 l} x_{u} & 0 \\
b_{21 u} y_{l} & 0 & r_{2 l}-\frac{b_{22 u}}{k_{2 u}} y_{u}+b_{21 u} x_{l}-q_{2} E & -\frac{b_{22 l}}{k_{2 l} l} y_{u} \\
0 & b_{211} y_{u} & r_{2 u}-\frac{b_{22 l}}{k_{2 l}} y_{l}+b_{21} x_{u}-q_{2} E
\end{array}\right) \text {, } \\
& \mathbf{V}_{2}=\left(\begin{array}{cccc}
r_{1 l}-\frac{b_{11 u}}{k_{1 u}} x_{u}-b_{12 u} y_{l}-q_{1} E & -\frac{b_{11 u}}{k_{1 u}} x_{l} & 0 & -b_{12 u} x_{l} \\
-\frac{b_{111}}{k_{1 l}} x_{u} & r_{1 u}-\frac{b_{11 l}}{k_{1 l}} x_{l}-b_{12 l} y_{u}-q_{1} E & -b_{12 l} x_{u} & 0 \\
b_{21 u} y_{u} & 0 & -\frac{b_{22 u}}{k_{2 u}} y_{u} & r_{2 l}-\frac{b_{22 u}}{k_{2 u}} y_{l}+b_{21 u} x_{l}-q_{2} E \\
0 & b_{211} y_{l} & r_{2 u}-\frac{b_{22 l}}{k_{2 l}} y_{u}+b_{21 l} x_{u}-q_{2} E & -\frac{b_{22}}{k_{2 l}} y_{l}
\end{array}\right) \text {, } \\
& \mathbf{V}_{3}=\left(\begin{array}{cccc}
-\frac{b_{11 u}}{k_{1 u}} x_{u} & r_{1 l}-\frac{b_{11 u}}{k_{1 u}} x_{l}-b_{12 u} y_{u}-q_{1} E & 0 & -b_{12 u} x_{u} \\
r_{1 u}-\frac{b_{11 l}}{k_{1 l}} x_{u}-b_{12 l} y_{l}-q_{1} E & -\frac{b_{11 l}}{k_{1 l}} x_{l} & -b_{12 l} x_{l} & 0 \\
b_{21 u} y_{l} & 0 & r_{2 l}-\frac{b_{22 u}}{k_{2 u}} y_{u}+b_{21 u} x_{l}-q_{2} E & -\frac{b_{22 l}}{k_{2 u}} y_{l} \\
0 & b_{21 l} y_{u} & -\frac{b_{2 l}}{k_{2 l}} y_{u} & r_{2 u}-\frac{b_{22 l}}{k_{2 l}} y_{l}+b_{21 l} x_{u}-q_{2} E
\end{array}\right) \text {, }
\end{aligned}
$$


and

$$
\mathbf{V}_{4}=\left(\begin{array}{cccc}
-\frac{b_{11 u}}{k_{1 u}} x_{u} & r_{1 l}-\frac{b_{11 u}}{k_{1 u}} x_{l}-b_{12 u} y_{u}-q_{1} E & 0 & -b_{12 u} x_{u} \\
r_{1 u}-\frac{b_{111}}{k_{1 l}} x_{u}-b_{12 l} y_{l}-q_{1} E & -\frac{b_{111}}{k_{11}} x_{l} & -b_{12 l} x_{l} & 0 \\
b_{21 u} y_{u} & 0 & -\frac{b_{22 u}}{k_{2 u}} y_{u} & r_{2 l}-\frac{b_{222 u}}{k_{2 u}} y_{l}+b_{21 u} x_{l}-q_{2} E \\
0 & b_{211} y_{l} & r_{2 u}-\frac{b_{22 l}}{k_{2 l}} y_{u}+b_{21} x_{u}-q_{2} E & -\frac{b_{22 l} l}{k_{2 l}} y_{l}
\end{array}\right) .
$$

The stability analysis of each aforementioned equilibrium is elaborated sequentially in the following section, pertaining to the harvesting parameter $E$.

\subsection{Eigenvalues of variational matrices}

\subsubsection{Trivial equilibrium points}

Calculating $\mathbf{V}_{1}, \mathbf{V}_{2}, \mathbf{V}_{3}$, and $\mathbf{V}_{4}$ at the corresponding trivial equilibrium points of the respective systems, the following eigenvalues are attained, respectively:

$$
\begin{aligned}
& \lambda_{1}\left(e_{10}\right)=r_{1 l}-q_{1} E, \quad \lambda_{2}\left(e_{10}\right)=r_{1 u}-q_{1} E, \\
& \lambda_{3}\left(e_{10}\right)=r_{2 l}-q_{2} E, \quad \lambda_{4}\left(e_{10}\right)=r_{2 u}-q_{2} E, \\
& \lambda_{1}\left(e_{20}\right)=\sqrt{\left(r_{2 l}-q_{2} E\right)\left(r_{2 u}-q_{2} E\right),} \quad \lambda_{2}\left(e_{20}\right)=r_{1 l}-q_{1} E, \quad \lambda_{3}\left(e_{20}\right)=r_{1 u}-q_{1} E, \\
& \lambda_{4}\left(e_{20}\right)=-\sqrt{\left(r_{2 l}-q_{2} E\right)\left(r_{2 u}-q_{2} E\right)}, \\
& \lambda_{1}\left(e_{30}\right)=-\sqrt{\left(r_{1 l}-q_{1} E\right)\left(r_{1 u}-q_{1} E\right)}, \quad \lambda_{2}\left(e_{30}\right)=r_{2 l}-q_{2} E, \quad \lambda_{3}\left(e_{30}\right)=r_{2 u}-q_{2} E, \\
& \lambda_{4}\left(e_{30}\right)=\sqrt{\left(r_{1 l}-q_{1} E\right)\left(r_{1 u}-q_{1} E\right)},
\end{aligned}
$$

and

$$
\begin{array}{ll}
\lambda_{1}\left(e_{40}\right)=-\sqrt{\left(r_{1 l}-q_{1} E\right)\left(r_{1 u}-q_{1} E\right)}, & \lambda_{2}\left(e_{40}\right)=-\sqrt{\left(r_{2 l}-q_{2} E\right)\left(r_{2 u}-q_{2} E\right)}, \\
\lambda_{3}\left(e_{40}\right)=\sqrt{\left(r_{1 l}-q_{1} E\right)\left(r_{1 u}-q_{1} E\right)}, & \lambda_{4}\left(e_{40}\right)=\sqrt{\left(r_{2 l}-q_{2} E\right)\left(r_{2 u}-q_{2} E\right)} .
\end{array}
$$

4.1.2 Axial equilibrium points

The eigenvalues of $\mathbf{V}_{1}\left(e_{11}\right)$ for system (3) are obtained as follows:

$$
\begin{aligned}
& \lambda_{1}\left(e_{11}\right)=-\sqrt{\left(r_{1 l}-q_{1} E\right)\left(r_{1 u}-q_{1} E\right)}, \\
& \lambda_{2}\left(e_{11}\right)=-\frac{b_{11 u}\left(r_{2 l}-q_{2} E\right)+b_{21 u} k_{1 u}\left(r_{1 l}-q_{1} E\right)}{b_{11 u}}, \\
& \lambda_{3}\left(e_{11}\right)=\sqrt{\left(r_{1 l}-q_{1} E\right)\left(r_{1 u}-q_{1} E\right)}, \quad \lambda_{4}\left(e_{11}\right)=-\frac{b_{11 l}\left(r_{2 u}-q_{2} E\right)+b_{21 l} k_{1 l}\left(r_{1 u}-q_{1} E\right)}{b_{11 l}} .
\end{aligned}
$$

From $\mathbf{V}_{2}\left(e_{21}\right)$ of system (4), we get the following characteristic equation:

$$
\lambda^{4}+\beta_{2} \lambda^{2}+\beta_{4}=0
$$

where

$$
\begin{aligned}
\beta_{1}= & 0, \\
\beta_{2}= & -\left(\frac{b_{11 l} k_{1 u}\left(r_{1 l}-q_{1} E\right)}{k_{1 l} b_{11 u}}\right)\left(\frac{b_{11 u} k_{1 l}\left(r_{1 u}-q_{1} E\right)}{k_{1 u} b_{11 l}}\right)\left(r_{2 l}-q_{2} E+\frac{b_{21 l} k_{1 u}\left(r_{1 l}-q_{1} E\right)}{k_{1 l} b_{11 u}}\right) \\
& \times\left(r_{2 u}-q_{2} E+\frac{b_{21 l} k_{1 l}\left(r_{1 u}-q_{1} E\right)}{k_{1 u} b_{11 l}}\right),
\end{aligned}
$$




$$
\begin{aligned}
\beta_{3}= & 0, \\
\beta_{4}= & \left(\frac{b_{11 l} k_{1 u}\left(r_{1 l}-q_{1} E\right)}{k_{1 l} b_{11 u}}\right)\left(\frac{b_{11 u} k_{1 l}\left(r_{1 u}-q_{1} E\right)}{k_{1 u} b_{11 l}}\right)\left(r_{2 l}-q_{2} E+\frac{b_{21 l} k_{1 u}\left(r_{1 l}-q_{1} E\right)}{k_{1 l} b_{11 u}}\right) \\
& \times\left(r_{2 u}-q_{2} E+\frac{b_{21 l} k_{1 l}\left(r_{1 u}-q_{1} E\right)}{k_{1 u} b_{11 l}}\right) .
\end{aligned}
$$

For system (5), calculating eigenvalues from $\mathbf{V}_{3}\left(e_{31}\right)$ at $e_{31}$, we get

$$
\begin{aligned}
& \lambda_{1}\left(e_{31}\right)=-\left(\frac{b_{11 u} k_{1 l}\left(r_{1 u}-q_{1} E\right)}{k_{1 u} b_{11 l}}\right), \quad \lambda_{2}\left(e_{31}\right)=-\left(\frac{b_{11 l} k_{1 u}\left(r_{1 l}-q_{1} E\right)}{k_{1 l} b_{11 u}}\right), \\
& \lambda_{3}\left(e_{31}\right)=\frac{b_{11 l}\left(r_{2 l}-q_{2} E\right)+b_{21 u} k_{1 l}\left(r_{1 u}-q_{1} E\right)}{b_{11 l}} \\
& \lambda_{4}\left(e_{31}\right)=\frac{b_{11 u}\left(r_{2 u}-q_{2} E\right)+b_{21 l} k_{1 u}\left(r_{1 l}-q_{1} E\right)}{b_{11 u}}
\end{aligned}
$$

and for system (6), the characteristic equation obtained from $\mathbf{V}_{4}\left(e_{41}\right)$ is

$$
\lambda^{4}+\beta_{1} \lambda^{3}+\beta_{2} \lambda^{2}+\beta_{3} \lambda+\beta_{4}=0
$$

where

$$
\begin{aligned}
\beta_{1}= & \frac{b_{11 l} k_{1 l}\left(q_{1} E-r_{1 u}\right)}{k_{1 u} b_{11 l}}+\frac{b_{11 l} k_{1 u}\left(q_{1} E-r_{1 l}\right)}{k_{1 l} b_{11 u}}, \\
\beta_{2}= & \left(\frac{b_{11 l} k_{1 u}\left(r_{1 l}-q_{1} E\right)}{k_{1 l} b_{11 u}}\right)\left(\frac{b_{11 u} k_{1 l}\left(r_{1 u}-q_{1} E\right)}{k_{1 u} b_{11 l}}\right) \\
& -\left(r_{2 l}-q_{2} E+\frac{b_{21 u} k_{1 u}\left(r_{1 l}-q_{1} E\right)}{b_{11 u}}\right)\left(r_{2 u}-q_{2} E+\frac{b_{21 l} k_{1 l}\left(r_{1 u}-q_{1} E\right)}{b_{11 l}}\right), \\
\beta_{3}= & \left(\frac{b_{11 l} k_{1 l}\left(q_{1} E-r_{1 u}\right)}{k_{1 u} b_{11 l}}+\frac{b_{11 l} k_{1 u}\left(q_{1} E-r_{1 l}\right)}{k_{1 l} b_{11 u}}\right)\left(r_{2 l}-q_{2} E+\frac{b_{21 u} k_{1 u}\left(r_{1 l}-q_{1} E\right)}{b_{11 u}}\right) \\
& \times\left(r_{2 u}-q_{2} E+\frac{b_{21 l} k_{1 l}\left(r_{1 u}-q_{1} E\right)}{b_{11 l}}\right), \\
\beta_{4}= & \left(\frac{b_{11 l} k_{1 l}\left(r_{1 u}-q_{1} E\right)}{k_{1 u} b_{11 l}}+\frac{b_{11 l} k_{1 u}\left(r_{1 l}-q_{1} E\right)}{k_{1 l} b_{11 u}}\right)\left(r_{2 l}-q_{2} E+\frac{b_{21 u} k_{1 u}\left(r_{1 l}-q_{1} E\right)}{b_{11 u}}\right) \\
& \times\left(r_{2 u}-q_{2} E+\frac{b_{21 l} k_{1 l}\left(r_{1 u}-q_{1} E\right)}{b_{11 l}}\right) .
\end{aligned}
$$

\subsubsection{Coexistence equilibrium points}

Furthermore, on simplifying $\mathbf{V}_{1}\left(e_{12}\right)$ for system (3), we get the characteristic equation

$$
\lambda^{4}+\beta_{2} \lambda^{2}+\beta_{4}=0,
$$

where

$$
\begin{aligned}
& \beta_{1}=0 \\
& \beta_{2}=-\left(\frac{b_{11 l} b_{11 u} x_{12 l} x_{12 u}}{k_{1 l} k_{1 u}}-b_{21 u} b_{12 l} x_{12 u} y_{12 l}+\frac{b_{22 l} b_{22 u} y_{12 l} y_{12 u}}{k_{2 l} k_{2 u}}-b_{21 l} b_{12 u} y_{12 u} x_{12 l}\right),
\end{aligned}
$$




$$
\begin{aligned}
& \beta_{3}=0, \\
& \beta_{4}=x_{12 l} x_{12 u} y_{12 l} y_{12 u}\left(\frac{b_{11 u} b_{22 u}}{k_{2 u}}+b_{21 u} b_{12 u}\right)\left(\frac{b_{22 l} b_{11 l}}{k_{1 l} k_{1 l}}+b_{21 l} b_{12 l}\right) .
\end{aligned}
$$

For system (4), the characteristic equation obtained from $\mathbf{V}_{2}\left(e_{22}\right)$ is

$$
\lambda^{4}+\beta_{1} \lambda^{3}+\beta_{2} \lambda^{2}+\beta_{3} \lambda+\beta_{4}=0
$$

where

$$
\begin{aligned}
\beta_{1}= & \frac{b_{22 u} y_{22 l}}{k_{2 u}}+\frac{b_{22 l} y_{22 u}}{k_{2 l}}, \\
\beta_{2}= & \frac{b_{22 u} b_{22 l} y_{22 l} y_{22 u}}{k_{2 u} k_{2 l}}-\frac{b_{11 u} b_{11 l} x_{22 l} x_{22 u}}{k_{1 u} k_{1 l}}, \\
\beta_{3}= & \left(\frac{b_{11 l} x_{22 l} y_{22 l} x_{22 u}}{k_{1 l}}\right)\left(\frac{b_{22 u} b_{11 u}}{k_{1 u} k_{2 u}}+b_{12 u} b_{21 u}\right) \\
& +\left(\frac{b_{11 u} x_{22 l} y_{22 u} x_{22 u}}{k_{1 u}}\right)\left(\frac{b_{22 l} b_{11 l}}{k_{1 l} k_{2 l}}+b_{12 l} b_{21 l}\right), \\
\beta_{4}= & -\frac{b_{11 u} x_{22 l}}{k_{1 u}}\left(\frac{b_{11 l} b_{22 u} b_{22 l} x_{22 u} y_{22 l} y_{22 u}}{k_{1 u} k_{2 u} k_{2 l}}+\frac{b_{12 l} b_{21 l} b_{22 u} x_{22 u} y_{22 u} y_{22 l}}{k_{2 u}}\right) \\
& -b_{12 u} x_{22 l}\left(\frac{b_{11 l} b_{21 u} b_{22 l} x_{22 u} y_{22 l} y_{22 u}}{k_{1 u} k_{2 l}}+b_{12 l} b_{21 l} b_{21 u} x_{22 u} y_{22 u} y_{22 l}\right) .
\end{aligned}
$$

The characteristic equation for system (5) obtained from $\mathbf{V}_{3}\left(e_{32}\right)$ is

$$
\lambda^{4}+\beta_{1} \lambda^{3}+\beta_{2} \lambda^{2}+\beta_{3} \lambda+\beta_{4}=0
$$

where

$$
\begin{aligned}
\beta_{1}= & \frac{b_{11 u} x_{32 l}}{k_{1 u}}+\frac{b_{11 l} x_{32 u}}{k_{2 l}}, \\
\beta_{2}= & \frac{b_{11 u} b_{11 l} x_{32 l} x_{32 u}}{k_{1 u} k_{1 l}}-\frac{b_{22 u} b_{22 l} y_{32 l} y_{32 u}}{k_{2 u} k_{2 l}}, \\
\beta_{3}= & \left(\frac{b_{22 l} x_{32 l} y_{32 l} x_{32 u}}{k_{2 l}}\right)\left(\frac{b_{22 u} b_{11 u}}{k_{1 u} k_{2 u}}+b_{12 u} b_{21 u}\right) \\
& +\left(\frac{b_{22 u} x_{32 l} y_{32 l} x_{32 u}}{k_{2 u}}\right)\left(\frac{b_{22 l} b_{11 l}}{k_{1 l} k_{2 l}}+b_{12 l} b_{21 l}\right), \\
\beta_{4}= & -\frac{b_{11 u} b_{22 u} x_{32 l} x_{32 u} y_{32 l} y_{32 u}}{k_{1 u} k_{2 u}}\left(\frac{b_{22 l} b_{11 l}}{k_{1 l} k_{2 l}}+b_{12 l} b_{21 l}\right) \\
& -b_{12 u} x_{32 l}\left(\frac{b_{11 l} x_{32 u}}{k_{1 l}} b_{21 u} y_{32 l} \frac{b_{22 l} y_{32 u}}{k_{2 l}}+b_{12 l} b_{21 l} b_{21 u} x_{32 u} y_{32 u} y_{32 l}\right)
\end{aligned}
$$

and the characteristic equation for system (6), generated from $\mathbf{V}_{4}\left(e_{42}\right)$, is

$$
\lambda^{4}+\beta_{1} \lambda^{3}+\beta_{2} \lambda^{2}+\beta_{3} \lambda+\beta_{4}=0
$$


where

$$
\begin{aligned}
\beta_{1}= & -\left(\frac{b_{11 u} x_{42 l}}{k_{1 u}}+\frac{b_{11 l} x_{42 u}}{k_{1 l}}+\frac{b_{22 u} y_{42 l}}{k_{2 u}}+\frac{b_{22 u} y_{42 u}}{k_{2 u}}\right), \\
\beta_{2}= & \frac{b_{11 u} x_{42 l}}{k_{1 u}} \frac{b_{11 l} x_{42 u}}{k_{1 l}}+\frac{b_{11 l} x_{42 u}}{k_{1 l}} \frac{b_{22 u} y_{42 l}}{k_{2 u}}+\frac{b_{22 u} y_{42 l}}{k_{2 u}} \frac{b_{22 l} y_{42 u}}{k_{2 u}} \\
& +\frac{b_{11 u} x_{42 l}}{k_{1 u}} \frac{b_{22 u} y_{42 l}}{k_{2 u}}-b_{12 u} x_{42 l} b_{12 u} y_{42 l} \\
& \times \frac{b_{11 u} x_{42 l}}{k_{1 u}} \frac{b_{22 l} y_{42 u}}{k_{2 l}}+\frac{b_{11 l} x_{42 u}}{k_{1 l}} \frac{b_{22 l} y_{42 u}}{k_{2 l}}+b_{12 l} x_{42 u} b_{12 l} y_{42 u}, \\
\beta_{3}= & \frac{b_{11 u} x_{42 l}}{k_{1 u}} \frac{b_{11 l} x_{42 u}}{k_{1 l}} \frac{b_{22 u} y_{42 l}}{k_{2 u}}+\frac{b_{11 l} x_{42 u}}{k_{1 l}} b_{12 u} x_{42 l} b_{12 u} y_{42 l} \\
& -\frac{b_{11 u} x_{42 l}}{k_{1 u}}\left(b_{12 l} x_{42 u} b_{12 l} y_{42 u}+\frac{b_{11 l} x_{42 u}}{k_{1 l}} \frac{b_{22 l} y_{42 u}}{k_{2 l}}\right) \\
& -\frac{b_{11 u} x_{42 l}}{k_{1 u}} \frac{b_{22 l} y_{42 u}}{k_{2 l}} \frac{b_{22 u} y_{42 l}}{k_{2 u}}-b_{12 u} x_{42 l} b_{12 u} y_{42 l} \frac{b_{22 l} y_{42 u}}{k_{2 l}} \\
& -\frac{b_{11 l} x_{42 u}}{k_{1 l}} \frac{b_{22 l} y_{42 u}}{k_{2 l}} \frac{b_{22 u} y_{42 l}}{k_{2 u}}-\frac{b_{22 u} y_{42 l}}{k_{2 u}} b_{12 l} x_{42 u} b_{12 l} y_{42 u}, \\
\beta_{4}= & \frac{b_{11 u} x_{42 l}}{k_{1 u}}\left(\frac{b_{11 l} x_{42 u}}{k_{1 l}} \frac{b_{22 l} y_{42 u}}{k_{2 l}} \frac{b_{22 u} y_{42 l}}{k_{2 u}}-\frac{b_{22 u} y_{42 l}}{k_{2 u}} b_{12 l} x_{42 u} b_{12 l} y_{42 u}\right) \\
& +b_{12 u} x_{42 l} b_{12 u} y_{42 l}\left(\frac{b_{11 l} x_{42 u}}{k_{1 l}} \frac{b_{22 l} y_{42 u}}{k_{2 l}}-b_{12 l} x_{42 u} b_{12 l} y_{42 u}\right) .
\end{aligned}
$$

\subsection{Stability conditions}

From the above mathematical discussion, we deduce the following results.

\section{Theorem 4.1}

(i) It can be clearly noticed that $\lambda_{i}\left(e_{20}\right)>0$ for $i=1,2,3, \lambda_{i}\left(e_{30}\right)>0$ for $i=2,3,4$, and $\lambda_{i}\left(e_{40}\right)>0$ for $i=3,4$. Thus, from the Routh-Hurwitz condition, $e_{20}, e_{30}$, and $e_{40}$ are unstable. The trivial equilibrium point $e_{10}$ for system (3) is stable, i.e., $\lambda_{i}\left(e_{10}\right)<0$ for $i=0,1,2,3,4$ only if

$$
E>\frac{r_{1 l}}{q_{1}}, \quad E>\frac{r_{1 u}}{q_{1}}, \quad E>\frac{r_{2 l}}{q_{2}}, \quad E>\frac{r_{2 u}}{q_{2}} .
$$

Hence, $e_{10}$ is locally asymptotically stable if $E>\max \left(\frac{r_{11}}{q_{1}}, \frac{r_{1 u}}{q_{1}}, \frac{r_{2 l}}{q_{2}}, \frac{r_{2 u}}{q_{2}}\right)$.

(ii) The axial equilibrium point $e_{11}$ of system (3) shows instability in view of the fact that $\lambda_{3}\left(e_{11}\right)>0, \lambda_{1}\left(e_{11}\right)<0, \lambda_{2}\left(e_{11}\right)<0$, and $\lambda_{4}\left(e_{11}\right)<0$. Additionally, $\beta_{1}=0, \beta_{3}=0$ for the axial equilibrium point $e_{21}$, hence from the Routh-Hurwitz condition $e_{21}$ is also not stable. On the other hand, $e_{31}$ becomes stable, i.e., $\lambda_{i}\left(e_{31}\right)<0$ for $i=3,4$, only if

$$
\begin{aligned}
& E>\frac{b_{11 l} r_{2 l}+b_{21 u} k_{1 l} r_{1 u}}{q_{2} b_{11 l}+q_{1} b_{21 l} k_{1 l}}, \quad E>\frac{b_{11 u} r_{2 u}+b_{21 l} k_{1 u} r_{1 l}}{q_{2} b_{11 u}+q_{1} b_{21 l} k_{1 u}}, \\
& E>\frac{r_{1 l}}{q_{1}}, \quad E>\frac{r_{1 u}}{q_{1}} .
\end{aligned}
$$


Hence, $e_{31}$ is locally asymptotically stable if

$$
E>\max \left(\frac{b_{11 l} r_{2 l}+b_{21 u} k_{1 l} r_{1 u}}{q_{2} b_{11 l}+q_{1} b_{21 l} k_{1 l}}, \frac{b_{11 u} r_{2 u}+b_{21 l} k_{1 u} r_{1 l}}{q_{2} b_{11 u}+q_{1} b_{21 l} k_{1 u}}, \frac{r_{1 l}}{q_{1}}, \frac{r_{1 u}}{q_{1}}\right)
$$

(iii) Correspondingly, an axial equilibrium point $e_{41}$ for system (6) is locally asymptotically stable, i.e., $\beta_{i}\left(e_{41}\right)>0$ for $i=0,1,2,3,4$, only if

$$
\begin{aligned}
& \frac{b_{11 l} k_{1 l}\left(q_{1} E-r_{1 u}\right)}{k_{1 u} b_{11 l}}+\frac{b_{11 l} k_{1 u}\left(q_{1} E-r_{1 l}\right)}{k_{1 l} b_{11 u}}>0, \\
& \left(\frac{b_{11 l} k_{1 u}\left(r_{1 l}-q_{1} E\right)}{k_{1 l} b_{11 u}}\right)\left(\frac{b_{11 u} k_{1 l}\left(r_{1 u}-q_{1} E\right)}{k_{1 u} b_{11 l}}\right) \\
& -\left(r_{2 l}-q_{2} E+\frac{b_{21 u} k_{1 u}\left(r_{1 l}-q_{1} E\right)}{b_{11 u}}\right)\left(r_{2 u}-q_{2} E+\frac{b_{21 l} k_{1 l}\left(r_{1 u}-q_{1} E\right)}{b_{11 l}}\right)>0, \\
& \left(\frac{b_{11 l} k_{1 l}\left(q_{1} E-r_{1 u}\right)}{k_{1 u} b_{11 l}}+\frac{b_{11 l} k_{1 u}\left(q_{1} E-r_{1 l}\right)}{k_{1 l} b_{11 u}}\right)\left(r_{2 l}-q_{2} E+\frac{b_{21 u} k_{1 u}\left(r_{1 l}-q_{1} E\right)}{b_{11 u}}\right) \\
& \quad \times\left(r_{2 u}-q_{2} E+\frac{b_{21 l} k_{1 l}\left(r_{1 u}-q_{1} E\right)}{b_{11 l}}\right)>0, \\
& \left(\frac{b_{11 l} k_{1 l}\left(r_{1 u}-q_{1} E\right)}{k_{1 u} b_{11 l}}+\frac{b_{11 l} k_{1 u}\left(r_{1 l}-q_{1} E\right)}{k_{1 l} b_{11 u}}\right)\left(r_{2 l}-q_{2} E+\frac{b_{21 u} k_{1 u}\left(r_{1 l}-q_{1} E\right)}{b_{11 u}}\right) \\
& \quad \times\left(r_{2 u}-q_{2} E+\frac{b_{21 l} k_{1 l}\left(r_{1 u}-q_{1} E\right)}{b_{11 l}}\right)>0,
\end{aligned}
$$

where, after some manipulation, expressions for $E$ can be established.

(iv) Evidently, the coexistence equilibrium point $e_{12}$ is not stable in view of the fact that $\beta_{1}=0, \beta_{3}=0$ of the corresponding characteristic equation. In the same way, $e_{22}, e_{32}$, and $e_{42}$ are unstable given that $\beta_{4}\left(e_{22}\right)<0, \beta_{4}\left(e_{32}\right)<0$, and $\beta_{1}\left(e_{42}\right)<0$, respectively.

\section{Methodological discussion}

\subsection{Implementation of fuzzy fractional Laplace transform}

To start with, the Caputo-type fractional operator on the left-hand side of each system (3)(6) is approximated by using fuzzy fractional Laplace transform [31, 34, 35]. Let $\mathbf{L}$ denote FFLT, then FFLT of Caputo-type fractional derivative of order $0<\sigma \leq 1$ of the functions $\tilde{x}(t)$ and $\tilde{y}(t)$ is expanded as follows:

$$
\mathbf{L}\left[{ }^{C} D_{t}^{\sigma} \tilde{x}(t)\right]=p^{\sigma} \mathbf{X}(p) \Theta p^{\sigma-1} \tilde{x}(0)
$$

if $\tilde{x}(t)$ is (i)-fgH-differentiable and

$$
\mathbf{L}\left[{ }^{C} D_{t}^{\sigma} \tilde{x}(t)\right]=-p^{\sigma-1} x(0) \Theta\left(-p^{\sigma} \mathbf{X}(p)\right)
$$

if $\tilde{x}(t)$ is (ii)-fgH-differentiable. Similarly,

$$
\begin{aligned}
& \mathbf{L}\left[{ }^{C} D_{t}^{\sigma} \tilde{y}(t)\right]=p^{\sigma} \mathbf{Y}(p) \Theta p^{\sigma-1} \tilde{y}(0), \\
& \mathbf{L}\left[{ }^{C} D_{t}^{\sigma} \tilde{y}(t)\right]=-p^{\sigma-1} \tilde{y}(0) \Theta\left(-p^{\sigma} \mathbf{Y}(p)\right)
\end{aligned}
$$


for (i)-fgH-differentiable and (ii)-fgH-differentiable of $\tilde{y}(t)$, respectively. Following the method of linearization $[34,35]$, we get the linearized form of $p^{\sigma}$ as follows:

$$
p^{\sigma} \approx \sigma p^{1}+(1-\sigma) p^{0}=\sigma p+(1-\sigma)
$$

On substitution of the above expansion of $p^{\sigma}$ in Eqs. (7)-(10) and employing the inverse FFLT, systems (3)-(6) convert into integer-order nonlinear differential equations, i.e., if both $\tilde{x}(t)$ and $\tilde{y}(t)$ are (i)-fgH-differentiable, then system (3) turns into

$$
\begin{aligned}
& \sigma \frac{d x_{l}(t ; \alpha)}{d t}+(1-\sigma)\left(x_{l}(t ; \alpha)-x_{u}(0 ; \alpha)\right) \\
& =x_{l}(t ; \alpha)\left(r_{1 l}(\alpha)-\frac{b_{11 u}(\alpha)}{k_{1 u}(\alpha)} x_{u}(t ; \alpha)-b_{12 u}(\alpha) y_{u}(t ; \alpha)-q_{1} E\right), \\
& \sigma \frac{d x_{u}(t ; \alpha)}{d t}+(1-\sigma)\left(x_{u}(t ; \alpha)-x_{l}(0 ; \alpha)\right) \\
& \quad=x_{u}(t ; \alpha)\left(r_{1 u}(\alpha)-\frac{b_{11 l}(\alpha)}{k_{1 l}(\alpha)} x_{l}(t ; \alpha)-b_{12 l}(\alpha) y_{l}(t ; \alpha)-q_{1} E\right), \\
& \sigma \frac{d y_{l}(t ; \alpha)}{d t}+(1-\sigma)\left(y_{l}(t ; \alpha)-y_{u}(0 ; \alpha)\right) \\
& \quad=y_{l}(t ; \alpha)\left(r_{2 l}(\alpha)-\frac{b_{22 u}(\alpha)}{k_{2 u}(\alpha)} y_{u}(t ; \alpha)+b_{21 l}(\alpha) x_{l}(t ; \alpha)-q_{2} E\right), \\
& \sigma \frac{d y_{u}(t ; \alpha)}{d t}+(1-\sigma)\left(y_{u}(t ; \alpha)-y_{l}(0 ; \alpha)\right) \\
& \quad=y_{u}(t ; \alpha)\left(r_{2 u}(\alpha)-\frac{b_{22 l}(\alpha)}{k_{2 l}(\alpha)} y_{l}(t ; \alpha)+b_{21 u}(\alpha) x_{u}(t ; \alpha)-q_{2} E\right)
\end{aligned}
$$

and if $\tilde{x}(t)$ is (i)-fgH-differentiable and $\tilde{y}(t)$ is (ii)-fgH-differentiable, then system (4) changes into

$$
\begin{aligned}
& \sigma \frac{d x_{l}(t ; \alpha)}{d t}+(1-\sigma)\left(x_{l}(t ; \alpha)-x_{u}(0 ; \alpha)\right) \\
& \quad=x_{l}(t ; \alpha)\left(r_{1 l}(\alpha)-\frac{b_{11 u}(\alpha)}{k_{1 u}(\alpha)} x_{u}(t ; \alpha)-b_{12 u}(\alpha) y_{l}(t ; \alpha)-q_{1} E\right), \\
& \sigma \frac{d x_{u}(t ; \alpha)}{d t}+(1-\sigma)\left(x_{u}(t ; \alpha)-x_{l}(0 ; \alpha)\right) \\
& \quad=x_{u}(t ; \alpha)\left(r_{1 u}(\alpha)-\frac{b_{11 l}(\alpha)}{k_{1 l}(\alpha)} x_{l}(t ; \alpha)-b_{12 l}(\alpha) y_{u}(t ; \alpha)-q_{1} E\right), \\
& \sigma \frac{d y_{l}(t ; \alpha)}{d t}+(1-\sigma)\left(y_{u}(t ; \alpha)-y_{u}(0 ; \alpha)\right) \\
& \quad=y_{u}(t ; \alpha)\left(r_{2 u}(\alpha)-\frac{b_{22 l}(\alpha)}{k_{2 l}(\alpha)} y_{l}(t ; \alpha)+b_{21 u}(\alpha) x_{u}(t ; \alpha)-q_{2} E\right), \\
& \sigma \frac{d y_{u}(t ; \alpha)}{d t}+(1-\sigma)\left(y_{l}(t ; \alpha)-y_{l}(0 ; \alpha)\right) \\
& \quad=y_{l}(t ; \alpha)\left(r_{2 l}(\alpha)-\frac{b_{22 u}(\alpha)}{k_{2 u}(\alpha)} y_{u}(t ; \alpha)+b_{21 l}(\alpha) x_{l}(t ; \alpha)-q_{2} E\right) .
\end{aligned}
$$


Similarly, if $\tilde{x}(t)$ is (ii)-fgH-differentiable and $\tilde{y}(t)$ is (i)-fgH-differentiable, then system (5) converts into

$$
\begin{aligned}
& \sigma \frac{d x_{l}(t ; \alpha)}{d t}+(1-\sigma)\left(x_{u}(t ; \alpha)-x_{u}(0 ; \alpha)\right) \\
& \quad=x_{u}(t ; \alpha)\left(r_{1 u}(\alpha)-\frac{b_{11 l}(\alpha)}{k_{1 l}(\alpha)} x_{l}(t ; \alpha)-b_{12 l}(\alpha) y_{l}(t ; \alpha)-q_{1} E\right) \\
& \sigma \frac{d x_{u}(t ; \alpha)}{d t}+(1-\sigma)\left(x_{l}(t ; \alpha)-x_{l}(0 ; \alpha)\right) \\
& \quad=x_{l}(t ; \alpha)\left(r_{1 l}(\alpha)-\frac{b_{11 u}(\alpha)}{k_{1 u}(\alpha)} x_{u}(t ; \alpha)-b_{12 u}(\alpha) y_{u}(t ; \alpha)-q_{1} E\right) \\
& \sigma \frac{d y_{l}(t ; \alpha)}{d t}+(1-\sigma)\left(y_{l}(t ; \alpha)-y_{u}(0 ; \alpha)\right) \\
& \quad=y_{l}(t ; \alpha)\left(r_{2 l}(\alpha)-\frac{b_{22 u}(\alpha)}{k_{2 u}(\alpha)} y_{u}(t ; \alpha)+b_{21 l}(\alpha) x_{u}(t ; \alpha)-q_{2} E\right) \\
& \sigma \frac{d y_{u}(t ; \alpha)}{d t}+(1-\sigma)\left(y_{u}(t ; \alpha)-y_{l}(0 ; \alpha)\right) \\
& \quad=y_{u}(t ; \alpha)\left(r_{2 u}(\alpha)-\frac{b_{22 l}(\alpha)}{k_{2 l}(\alpha)} y_{l}(t ; \alpha)+b_{21 u}(\alpha) x_{l}(t ; \alpha)-q_{2} E\right)
\end{aligned}
$$

and if both $\tilde{x}(t)$ and $\tilde{y}(t)$ are (ii)-fgH-differentiable, then system (6) becomes

$$
\begin{aligned}
& \sigma \frac{d x_{l}(t ; \alpha)}{d t}+(1-\sigma)\left(x_{u}(t ; \alpha)-x_{u}(0 ; \alpha)\right) \\
& \quad=x_{u}(t ; \alpha)\left(r_{1 u}(\alpha)-\frac{b_{11 l}(\alpha)}{k_{1 l}(\alpha)} x_{l}(t ; \alpha)-b_{12 l}(\alpha) y_{u}(t ; \alpha)-q_{1} E\right), \\
& \sigma \frac{d x_{u}(t ; \alpha)}{d t}+(1-\sigma)\left(x_{l}(t ; \alpha)-x_{l}(0 ; \alpha)\right) \\
& \quad=x_{l}(t ; \alpha)\left(r_{1 l}(\alpha)-\frac{b_{11 l}(\alpha)}{k_{1 l}(\alpha)} x_{l}(t ; \alpha)-b_{12 u}(\alpha) y_{l}(t ; \alpha)-q_{1} E\right), \\
& \sigma \frac{d y_{l}(t ; \alpha)}{d t}+(1-\sigma)\left(y_{u}(t ; \alpha)-y_{u}(0 ; \alpha)\right) \\
& \quad=y_{u}(t ; \alpha)\left(r_{2 u}(\alpha)-\frac{b_{22 l}(\alpha)}{k_{2 l}(\alpha)} y_{l}(t ; \alpha)+b_{21 u}(\alpha) x_{u}(t ; \alpha)-q_{2} E\right), \\
& \sigma \frac{d y_{u}(t ; \alpha)}{d t}+(1-\sigma)\left(y_{l}(t ; \alpha)-y_{l}(0 ; \alpha)\right) \\
& \quad=y_{l}(t ; \alpha)\left(r_{2 l}(\alpha)-\frac{b_{22 u}(\alpha)}{k_{2 u}(\alpha)} y_{u}(t ; \alpha)+b_{21 l}(\alpha) x_{l}(t ; \alpha)-q_{2} E\right)
\end{aligned}
$$

with the same initial conditions as for system (2).

\subsection{Numerical illustration}

Considering time $t$ in years and the population of species in billions, the experiment is carried out for some assumed numerical values of the parameters. The numerical equilibrium points of systems (12)-(15) for fractional indices $\sigma=0.5,0.75,0.95,1$, fuzzy parameter $\alpha=$ 0.5 , ecological parameters $q_{1}=0.2, q_{2}=0.5, E=11, \tilde{r}_{1}=(2.5,2.6,2.7), \tilde{r}_{2}=(3,3.3,3.5), \tilde{b}_{11}=$ 
$(0.2,0.3,0.6), \tilde{b}_{12}=(0.3,0.6,0.7), \tilde{b}_{21}=(0.1,0.3,0.5), \tilde{b}_{22}=(0.6,0.8,0.9), \tilde{k}_{1}=(50,55,60)$, $\tilde{k}_{2}=(30,35,40)$, and initial conditions $\tilde{x}_{0}=(0.3,0.5,0.9)$ and $\tilde{y}_{0}=(0.4,0.5,0.7)$ are shown in Table 1. It interprets the constant population densities of prey and predator with respect to fractional variation in time. The elaborations include the perceptions of fgHdifferentiability, i.e., fractional variation in either increasing or decreasing growth rate patterns of the species. For instance, some environmental factors or economical activities that affect the survival of the species may precipitously cause the growth to be either in increasing or decreasing motion. Furthermore, Tables $2-5$ ratify stability and originality of

Table 1 Numerical equilibrium points for different values of the fractional index $\sigma$ and $\alpha=0.5$

\begin{tabular}{lllll}
\hline & $\sigma=0.5$ & $\sigma=0.75$ & $\sigma=0.95$ & $\sigma=1$ \\
\hline System (12) & $(19.8,8.3,0,0)$ & $(45.5,15.7,0,0)$ & $(84.9,5.2,0,0.4)$ & $(0,0,0,0)$ \\
& $(1.4,1.8,0.1,0.1)$ & $(10.8,16.9,0.3,0)$ & $(84.3,38.5,0,0)$ & $(94.5,44.7,0,0)$ \\
& $(0,7.9,0,0)$ & $(11.1,3.8,0.4,0.1)$ & $(11.8,5.3,0.8,0.4)$ & $(11.8,5.3,0.9,0.5)$ \\
System (13) & $(1.6,1.6,0.1,0.1)$ & $(8.5,4.9,0.1,0.4)$ & $(11.7,0,0.5,0)$ & $(0,0,0,0)$ \\
& $(1.1,14.6,0.1,0)$ & $(47.5,14.6,0,0)$ & $(84.3,38.5,0,0)$ & $(94.5,44.7,0,0)$ \\
& $(19.5,0.9,0,0.1)$ & $(0,5.2,0,0.5)$ & $(11.8,5.3,0.4,0.8)$ & $(11.8,5.3,0.5,0.9)$ \\
System (14) & $(0.84,3.1,0.1,0.1)$ & $(2.4,11.4,0.4,0)$ & $(0,11.8,0.8,0)$ & $(0,0,0,0)$ \\
& $(9.01,2.3,0.1,0)$ & & $(84.7,38.4,0,0)$ & $(94.5,44.7,0,0)$ \\
& $(0.4,30.8,0,0.1)$ & & $(5.2,11.8,0.8,0.3)$ & $(5.3,11.8,0.9,0.4)$ \\
System (15) & $(0.9,2.5,0.1,0.2)$ & $(8.05,5.02,0.1,0.4)$ & $(0,5.2,0,0.9)$ & $(0,0,0,0)$ \\
& $(0.6,16.9,0.1,0)$ & $(48.7,14.3,0,0)$ & $(84.3,38.5,0,0)$ & $(94.5,44.7,0,0)$ \\
& $(19.1,1.7,0,0.2)$ & $(9.9,0,0.2,0)$ & $(11.8,5.3,0.4,0.8)$ & $(11.8,5.3,0.5,0.9)$ \\
\hline
\end{tabular}

Table 2 Solutions of system (12) with the comparison to GL and ABM for $\sigma=0.95, \alpha=0.6$

\begin{tabular}{|c|c|c|c|c|c|c|}
\hline \multirow[t]{2}{*}{$t$} & \multicolumn{2}{|l|}{ LFFRK } & \multicolumn{2}{|l|}{ GL [15] } & \multicolumn{2}{|l|}{ ABM [38] } \\
\hline & $\overline{[\tilde{x}(t)]^{\alpha}}$ & {$[\tilde{y}(t)]^{\alpha}$} & $\overline{[\tilde{x}(t)]^{\alpha}}$ & {$[\tilde{y}(t)]^{\alpha}$} & {$[\tilde{x}(t)]^{\alpha}$} & {$[\tilde{y}(t)]^{\alpha}$} \\
\hline 0.0 & {$[0.4200,0.6600]$} & {$[0.4600,0.5800]$} & {$[0.4200,0.6600]$} & {$[0.4600,0.5800]$} & {$[0.4200,0.6600]$} & {$[0.4600,0.5800]$} \\
\hline 0.1 & {$[0.4042,0.6362]$} & {$[0.4689,0.5969]$} & {$[0.4025,0.6371]$} & {$[0.4694,0.5996]$} & {$[0.4016,0.6324]$} & {$[0.4704,0.5995]$} \\
\hline 0.2 & {$[0.3888,0.6131]$} & {$[0.4777,0.6136]$} & {$[0.3859,0.6152]$} & {$[0.4777,0.6175]$} & {$[0.3838,0.6057]$} & {$[0.4805,0.6186]$} \\
\hline 0.3 & {$[0.3738,0.5907]$} & {$[0.4863,0.6300]$} & {$[0.3701,0.5944]$} & {$[0.4855,0.6347]$} & {$[0.3667,0.5799]$} & {$[0.4903,0.6375]$} \\
\hline 0.4 & {$[0.3593,0.5690]$} & {$[0.4946,0.6462]$} & {$[0.3549,0.5744]$} & {$[0.4929,0.6515]$} & {$[0.3501,0.5551]$} & {$[0.4999,0.6559]$} \\
\hline 0.5 & {$[0.3453,0.5480]$} & {$[0.5027,0.6620]$} & {$[0.3402,0.5552]$} & {$[0.4999,0.6679]$} & {$[0.3341,0.5311]$} & {$[0.5091,0.6741]$} \\
\hline 0.6 & {$[0.3317,0.5277]$} & {$[0.5106,0.6776]$} & {$[0.3262,0.5366]$} & {$[0.5068,0.6841]$} & {$[0.3187,0.5081]$} & {$[0.5181,0.6918]$} \\
\hline 0.7 & {$[0.3186,0.5081]$} & {$[0.5183,0.6929]$} & {$[0.3126,0.5188]$} & {$[0.5133,0.6999]$} & {$[0.3039,0.4859]$} & {$[0.5267,0.7091]$} \\
\hline 0.8 & {$[0.3059,0.4890]$} & {$[0.5257,0.7078]$} & {$[0.2996,0.5015]$} & {$[0.5196,0.7154]$} & {$[0.2898,0.4647]$} & {$[0.5350,0.7259]$} \\
\hline 0.9 & {$[0.2937,0.4707]$} & {$[0.5329,0.7224]$} & {$[0.2869,0.4849]$} & {$[0.5257,0.7306]$} & {$[0.2762,0.4443]$} & {$[0.5431,0.7422]$} \\
\hline 1.0 & {$[0.2819,0.4529]$} & {$[0.5399,0.7366]$} & {$[0.2748,0.4688]$} & {$[0.5315,0.7455]$} & {$[0.2632,0.4247]$} & {$[0.5508,0.7581]$} \\
\hline
\end{tabular}

Table 3 Solutions of system (13) with the comparison to GL and ABM for $\sigma=0.95, \alpha=0.6$

\begin{tabular}{|c|c|c|c|c|c|c|}
\hline \multirow[t]{2}{*}{ t } & \multicolumn{2}{|l|}{ LFFRK } & \multicolumn{2}{|l|}{ GL [15] } & \multicolumn{2}{|l|}{ ABM [38] } \\
\hline & {$[\tilde{x}(t)]^{\alpha}$} & {$[\tilde{y}(t)]^{\alpha}$} & {$[\tilde{x}(t)]^{\alpha}$} & {$[\tilde{y}(t)]^{\alpha}$} & {$[\tilde{x}(t)]^{\alpha}$} & {$[\tilde{y}(t)]^{\alpha}$} \\
\hline 0.0 & {$[0.4200,0.6600]$} & {$[0.4600,0.5800]$} & {$[0.4200,0.6600]$} & {$[0.4600,0.5800]$} & {$[0.4200,0.6600]$} & {$[0.4600,0.5800]$} \\
\hline 0.1 & {$[0.4064,0.6329]$} & {$[0.4774,0.5884]$} & {$[0.4048,0.6337]$} & {$[0.4789,0.5898]$} & {$[0.4042,0.6284]$} & {$[0.4802,0.5897]$} \\
\hline 0.2 & {$[0.3929,0.6068]$} & {$[0.4943,0.5968]$} & {$[0.3901,0.6088]$} & {$[0.4961,0.5985]$} & {$[0.3888,0.5982]$} & {$[0.4997,0.5993]$} \\
\hline 0.3 & {$[0.3798,0.5817]$} & {$[0.5106,0.6050]$} & {$[0.3761,0.5853]$} & {$[0.5123,0.6069]$} & {$[0.3737,0.5692]$} & {$[0.5185,0.6087]$} \\
\hline 0.4 & {$[0.3670,0.5575]$} & {$[0.5265,0.6132]$} & {$[0.3625,0.5628]$} & {$[0.5278,0.6151]$} & {$[0.3590,0.5415]$} & {$[0.5367,0.6181]$} \\
\hline 0.5 & {$[0.3545,0.5342]$} & {$[0.5419,0.6212]$} & {$[0.3493,0.5413]$} & {$[0.5427,0.6230]$} & {$[0.3447,0.5151]$} & {$[0.5542,0.6273]$} \\
\hline 0.6 & {$[0.3423,0.5118]$} & {$[0.5568,0.6291]$} & {$[0.3366,0.5208]$} & {$[0.5571,0.6308]$} & {$[0.3309,0.4898]$} & {$[0.5711,0.6363]$} \\
\hline 0.7 & {$[0.3304,0.4903]$} & {$[0.5712,0.6369]$} & {$[0.3243,0.5009]$} & {$[0.5709,0.6384]$} & {$[0.3175,0.4657]$} & {$[0.5873,0.6451]$} \\
\hline 0.8 & {$[0.3189,0.4696]$} & {$[0.5852,0.6445]$} & {$[0.3123,0.4820]$} & {$[0.5843,0.6458]$} & {$[0.3045,0.4427]$} & {$[0.6029,0.6536]$} \\
\hline 0.9 & {$[0.3077,0.4497]$} & {$[0.5987,0.6519]$} & {$[0.3008,0.4638]$} & {$[0.5972,0.6531]$} & {$[0.2919,0.4207]$} & {$[0.6179,0.6619]$} \\
\hline 1.0 & {$[0.2968,0.4307]$} & {$[0.6117,0.6591]$} & {$[0.2896,0.4462]$} & {$[0.6097,0.6602]$} & {$[0.2799,0.3998]$} & {$[0.6322,0.6701]$} \\
\hline
\end{tabular}


Table 4 Solutions of system (14) with the comparison to GL and ABM for $\sigma=0.95, \alpha=0.6$

\begin{tabular}{|c|c|c|c|c|c|c|}
\hline \multirow[t]{2}{*}{ t } & \multicolumn{2}{|l|}{ LFFRK } & \multicolumn{2}{|l|}{ GL [15] } & \multicolumn{2}{|l|}{ ABM [38] } \\
\hline & {$[\tilde{x}(t)]^{\alpha}$} & {$[\tilde{y}(t)]^{\alpha}$} & $\overline{[x(t)]^{\alpha}}$ & {$[\tilde{y}(t)]^{\alpha}$} & {$[\tilde{x}(t)]^{\alpha}$} & {$[\tilde{y}(t)]^{\alpha}$} \\
\hline 0.0 & {$[0.4200,0.6600]$} & {$[0.4600,0.5800]$} & {$[0.4200,0.6600]$} & {$[0.4600,0.5800]$} & {$[0.4200,0.6600]$} & {$[0.4600,0.5800]$} \\
\hline 0.1 & {$[0.3973,0.6431]$} & {$[0.4738,0.5905]$} & {$[0.3964,0.6432]$} & {$[0.4748,0.5925]$} & {$[0.3938,0.6402]$} & {$[0.4759,0.5923]$} \\
\hline 0.2 & {$[0.3748,0.6271]$} & {$[0.4877,0.6006]$} & {$[0.3737,0.6275]$} & {$[0.4885,0.6033]$} & {$[0.3680,0.6215]$} & {$[0.4917,0.6039]$} \\
\hline 0.3 & {$[0.3526,0.6121]$} & {$[0.5015,0.6101]$} & {$[0.3517,0.6128]$} & {$[0.5017,0.6133]$} & {$[0.3426,0.6040]$} & {$[0.5075,0.6151]$} \\
\hline 0.4 & {$[0.3307,0.5979]$} & {$[0.5153,0.6192]$} & {$[0.3302,0.5992]$} & {$[0.5148,0.6227]$} & {$[0.3176,0.5877]$} & {$[0.5232,0.6254]$} \\
\hline 0.5 & {$[0.3090,0.5846]$} & {$[0.5290,0.6277]$} & {$[0.3092,0.5865]$} & {$[0.5278,0.6314]$} & {$[0.2929,0.5726]$} & {$[0.5389,0.6352]$} \\
\hline 0.6 & {$[0.2876,0.5723]$} & {$[0.5428,0.6357]$} & {$[0.2884,0.5747]$} & {$[0.5406,0.6396]$} & {$[0.2685,0.5588]$} & {$[0.5546,0.6442]$} \\
\hline 0.7 & {$[0.2662,0.5609]$} & {$[0.5565,0.6431]$} & {$[0.2679,0.5638]$} & {$[0.5535,0.6473]$} & {$[0.2443,0.5461]$} & {$[0.5702,0.6525]$} \\
\hline 0.8 & {$[0.2451,0.5505]$} & {$[0.5702,0.6501]$} & {$[0.2477,0.5538]$} & {$[0.5666,0.6543]$} & {$[0.2204,0.5348]$} & {$[0.5858,0.6601]$} \\
\hline 0.9 & {$[0.2241,0.5410]$} & {$[0.5839,0.6564]$} & {$[0.2277,0.5446]$} & {$[0.5791,0.6608]$} & {$[0.1966,0.5247]$} & {$[0.6014,0.6669]$} \\
\hline 1.0 & {$[0.2031,0.5325]$} & {$[0.5977,0.6621]$} & {$[0.2078,0.5363]$} & {$[0.5919,0.6668]$} & {$[0.1730,0.5159]$} & {$[0.6170,0.6729]$} \\
\hline
\end{tabular}

Table 5 Solutions of system (15) with the comparison to $\mathrm{GL}$ and ABM for $\sigma=0.95, \alpha=0.6$

\begin{tabular}{|c|c|c|c|c|c|c|}
\hline \multirow[t]{2}{*}{$t$} & \multicolumn{2}{|l|}{ LFFRK } & \multicolumn{2}{|l|}{ GL [15] } & \multicolumn{2}{|l|}{ ABM [38] } \\
\hline & $\overline{[\tilde{x}(t)]^{\alpha}}$ & {$[\tilde{y}(t)]^{\alpha}$} & $\overline{[\tilde{x}(t)]^{\alpha}}$ & {$[\tilde{y}(t)]^{\alpha}$} & $\overline{[\tilde{x}(t)]^{\alpha}}$ & {$[\tilde{y}(t)]^{\alpha}$} \\
\hline 0.0 & {$[0.4200,0.6600]$} & {$[0.4600,0.5800]$} & {$[0.4200,0.6600]$} & {$[0.4600,0.5800]$} & {$[0.4200,0.6600]$} & {$[0.4600,0.5800]$} \\
\hline 0.1 & {$[0.3939,0.6453]$} & {$[0.4775,0.5883]$} & {$[0.3929,0.6455]$} & {$[0.4793,0.5897]$} & {$[0.3899,0.6428]$} & {$[0.4801,0.5897]$} \\
\hline 0.2 & {$[0.3682,0.6315]$} & {$[0.4949,0.5962]$} & {$[0.3670,0.6318]$} & {$[0.4971,0.5978]$} & {$[0.3603,0.6266]$} & {$[0.5001,0.5989]$} \\
\hline 0.3 & {$[0.3429,0.6184]$} & {$[0.5121,0.6038]$} & {$[0.3421,0.6191]$} & {$[0.5142,0.6054]$} & {$[0.3313,0.6114]$} & {$[0.5198,0.6077]$} \\
\hline 0.4 & {$[0.3181,0.6061]$} & {$[0.5291,0.6111]$} & {$[0.3178,0.6072]$} & {$[0.5308,0.6126]$} & {$[0.3029,0.5974]$} & {$[0.5393,0.6159]$} \\
\hline 0.5 & {$[0.2936,0.5947]$} & {$[0.5459,0.6178]$} & {$[0.2942,0.5962]$} & {$[0.5472,0.6193]$} & {$[0.2750,0.5844]$} & {$[0.5586,0.6237]$} \\
\hline 0.6 & {$[0.2695,0.5842]$} & {$[0.5627,0.6242]$} & {$[0.2710,0.5859]$} & {$[0.5632,0.6256]$} & {$[0.2476,0.5727]$} & {$[0.5776,0.6308]$} \\
\hline 0.7 & {$[0.2457,0.5746]$} & {$[0.5792,0.6301]$} & {$[0.2483,0.5766]$} & {$[0.5790,0.6314]$} & {$[0.2206,0.5621]$} & {$[0.5964,0.6373]$} \\
\hline 0.8 & {$[0.2222,0.5659]$} & {$[0.5956,0.6354]$} & {$[0.2259,0.5680]$} & {$[0.5946,0.6368]$} & {$[0.1939,0.5527]$} & {$[0.6149,0.6431]$} \\
\hline 0.9 & {$[0.1989,0.5580]$} & {$[0.6118,0.6403]$} & {$[0.2040,0.5602]$} & {$[0.6100,0.6417]$} & {$[0.1677,0.5446]$} & {$[0.6334,0.6482]$} \\
\hline 1.0 & {$[0.1759,0.5513]$} & {$[0.6279,0.6445]$} & {$[0.1823,0.5533]$} & {$[0.6252,0.6462]$} & {$[0.1418,0.5378]$} & {$[0.6517,0.6525]$} \\
\hline
\end{tabular}

the numerical solutions obtained by utilizing the well-known methods, namely FFRK, GL [15], and ABM [38]. These solutions are computed, by considering $\sigma=0.95, \alpha=0.6, q_{1}=$ $0.02, q_{2}=0.001, E=7, \tilde{r}_{1}=(0.001,0.002,0.003), \tilde{r}_{2}=(0.009,0.01,0.02), \tilde{b}_{11}=(0.2,0.4,0.6)$, $\tilde{b}_{12}=(0.4,0.42,0.44), \tilde{b}_{21}=(0.4,0.42,0.44), \tilde{b}_{22}=(0.09,0.1,0.3), \tilde{k}_{1}=(53,65,69)$, and $\tilde{k}_{2}=$ $(74,80,85)$ with the same initial conditions as defined above. Evidently, the solutions of systems from GL and ABM substantiate the results achieved from the FFRK method up to two to three decimal places.

In addition, all the graphical solutions of systems (12)-(15) are acquired by using the FFRK method of Mathematica 11, taking $\tilde{r}_{1}=(2.5,2.6,2.7), \tilde{r}_{2}=(3,3.3,3.5), \tilde{b}_{11}=$ $(0.2,0.3,0.6), \tilde{b}_{12}=(0.3,0.6,0.7), \tilde{b}_{21}=(0.1,0.3,0.5), \tilde{b}_{22}=(0.6,0.8,0.9), \tilde{k}_{1}=(50,55,60)$, $\tilde{k}_{2}=(30,35,40)$. As the parameters are considered to be triangular fuzzy numbers, we also depict all the possible values of these parameters graphically in Figs. 1(a)-(d), which can be assumed in the governing case. Figs $2-5$ illustrate the effect of harvesting threshold $E$ on the population growth of $\tilde{x}(t)$ and $\tilde{y}(t)$ for some cases of catchability parameters of prey and predator, i.e., $q_{1}$ and $q_{2}$ at $\alpha=0.98, \sigma=0.998$, and $t \in[0,40]$. It is observed from Figs. 2(a)-(b), plotted for system (12), when $q_{1}=q_{2}$, the population of the predator $y_{l}(t ; \alpha)$ undergoes some oscillations when $E=2,3$ and gradually becomes zero after some time. However, the population of prey $x_{l}(t ; \alpha)$ produces a minor curvature, and after some time it tends to zero when $E \geq 4$, which validates the stability condition $E>\max \left(\frac{r_{1 l}}{q_{1}}, \frac{r_{1 u}}{q_{1}}, \frac{r_{2 l}}{q_{2}}, \frac{r_{2 u}}{q_{2}}\right)$. In case of $q_{1}>q_{2}$ and $q_{1}<q_{2}$, as shown in Figs. 2(c)-(d) and 2(e)- 


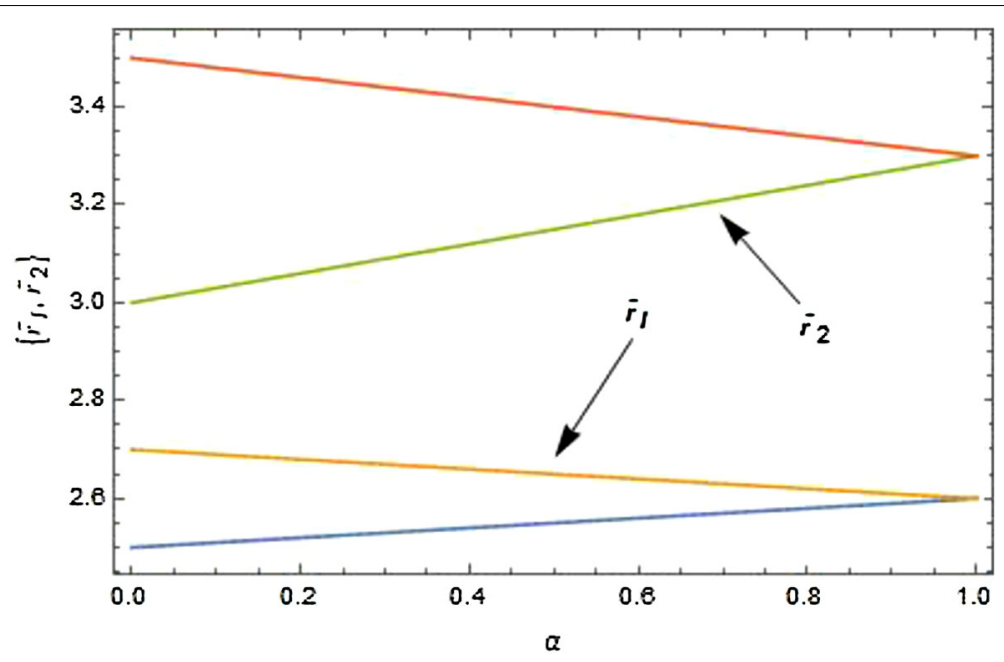

(a) Estimated values for $\tilde{r}_{1}$ and $\tilde{r}_{2}$ with membership degrees $\alpha$

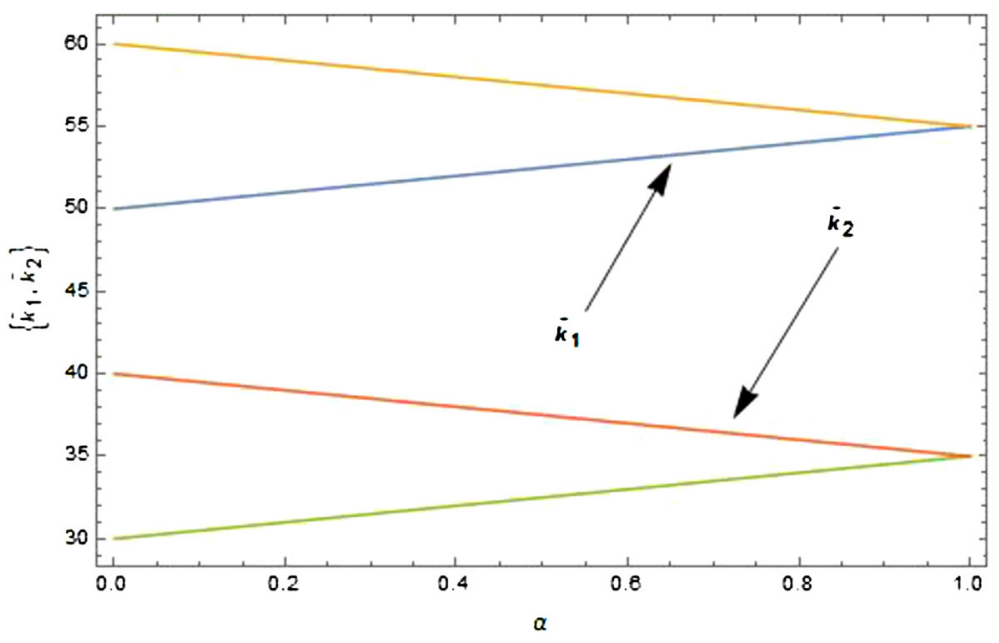

(b) Estimated values for $\tilde{k}_{1}$ and $\tilde{k}_{2}$ with membership degrees $\alpha$

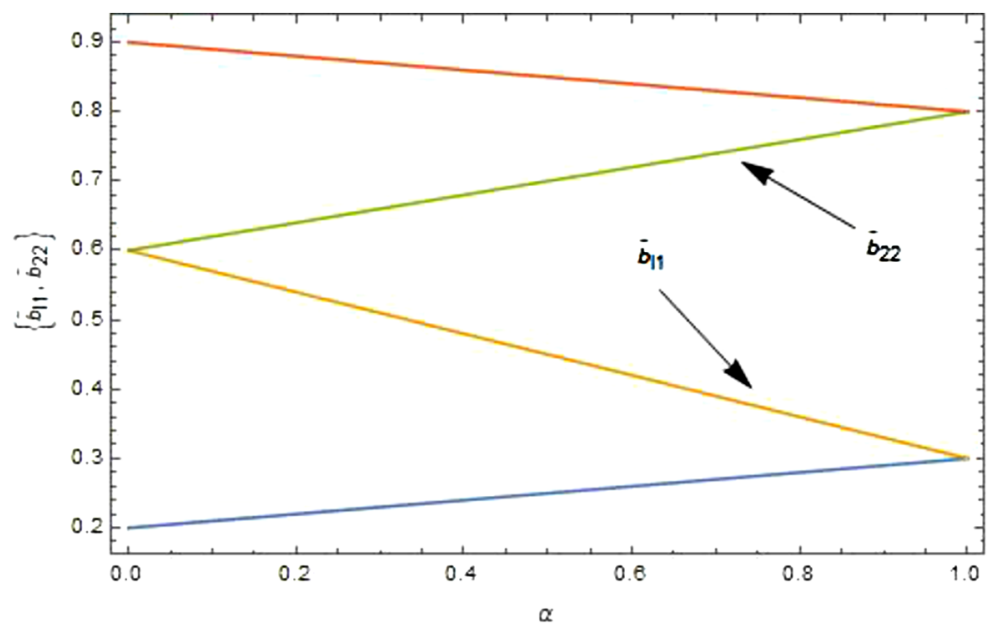

(c) Estimated values for $\tilde{b}_{11}$ and $\tilde{b}_{22}$ with membership degrees $\alpha$

Figure 1 Triangular fuzzy structures of biological parameters for systems (12)-(15) 


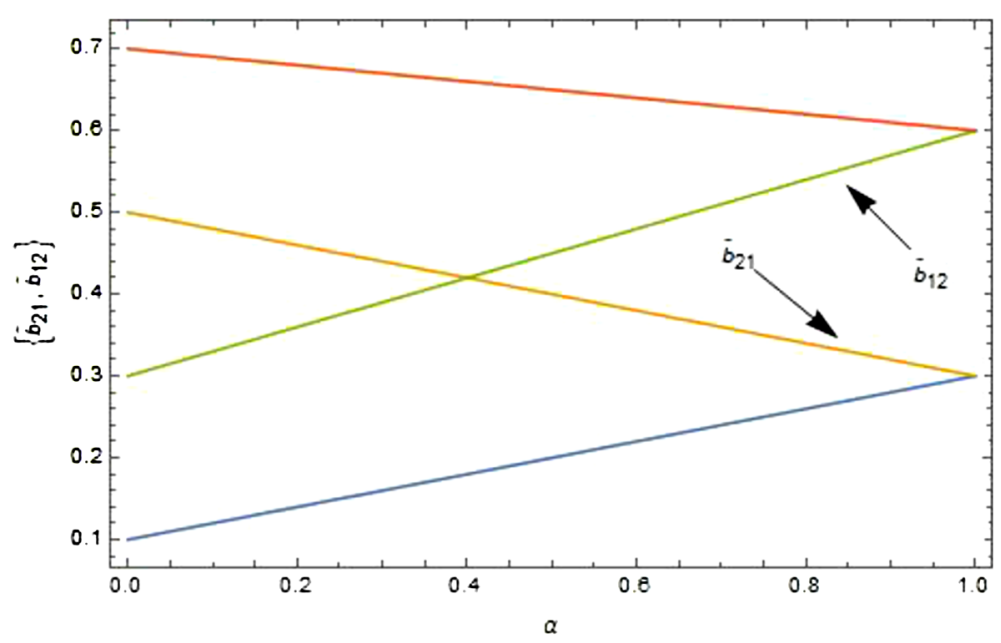

(d) Estimated values for $\tilde{b}_{21}$ and $\tilde{b}_{12}$ with membership degrees $\alpha$

Figure 1 Continued

(f), respectively, the trivial stability is found only when $E=5$ and oscillatory dynamics are generated elsewhere. Figs. 3(a)-(d) describe the axial equilibrium points of system (13), i.e., existence of predator $\tilde{y}(t)$ and extinction of prey $\tilde{x}(t)$ when $q_{1}=q_{2}$ and $q_{1}>q_{2}$. However, when catchability of prey becomes less than that of predators as presented in Figs. 3(e)-(f), oscillations of prey are observed when $E \geq 4$. System (14) shows trivial stability for all the cases of catchability only when $E=5$, as portrayed in Figs. 4(a)-(f), while unstable for other values of $E$. Relatedly, Figs. 5(a)-(f), plotted for system (15), are also unstable, except for $E=5$. From this analysis of harvesting parameters in conjunction with the catchability of the species, it can be interestingly remarked that the population of both the species, $\tilde{x}(t)$ and $\tilde{y}(t)$, goes to extinction if the harvesting rate $E$ is greater than the ratio of logistic growth and catchability coefficients. The population of both the species, $\tilde{x}(t)$ and $\tilde{y}(t)$, becomes stable if the harvesting rate $E$ of both the species is less than the ratio of imprecise biological parameters $\tilde{r}_{1}, \tilde{r}_{2}, \tilde{b}_{11}, \tilde{b}_{12}, \tilde{b}_{21}, \tilde{b}_{22}, \tilde{k}_{1}$, and $\tilde{k}_{2}$, as $\alpha$ increases.

In addition to that, we also carried out the existence analysis of the limit cycles of systems (12) -(15) through the phase planes, pictured in Figs. 6(a)-(d) and 7(a)-(d) for $\alpha=1, q_{1}=$ $0.2, q_{2}=0.7, E=8$, and at different values of $\sigma$. From each curve the existence of the limit cycles for prey and predator can be clearly interpreted to be in the intervals $3 \leq \tilde{x}^{*}(t) \leq 30$ and $0.5 \leq \tilde{y}^{*}(t) \leq 10$, accordingly, in the time interval $t \in[0,10]$. Besides, we also induce the limit cycles of systems (12)-(15) in Figs. 8(a)-(d) for $\alpha=1, q_{1}=0.2, q_{2}=0.7$, and $E=8$ for $t \in[0,20]$. These plots are the novel source of dynamical study of ecological theory, as they illustratively define the historical behavior of the population growth in an imprecise environment.

Each system delivers distinctive dynamics of $\tilde{x}(t)$ and $\tilde{y}(t)$ with different conditions of the harvesting parameter. Including a brief comparison between the cases of fgHdifferentiability, it is found that systems (12), (14), and (15) interpret the stability of the trivial equilibrium point; however, system (13) deduces the stability of axial equilibrium point. 


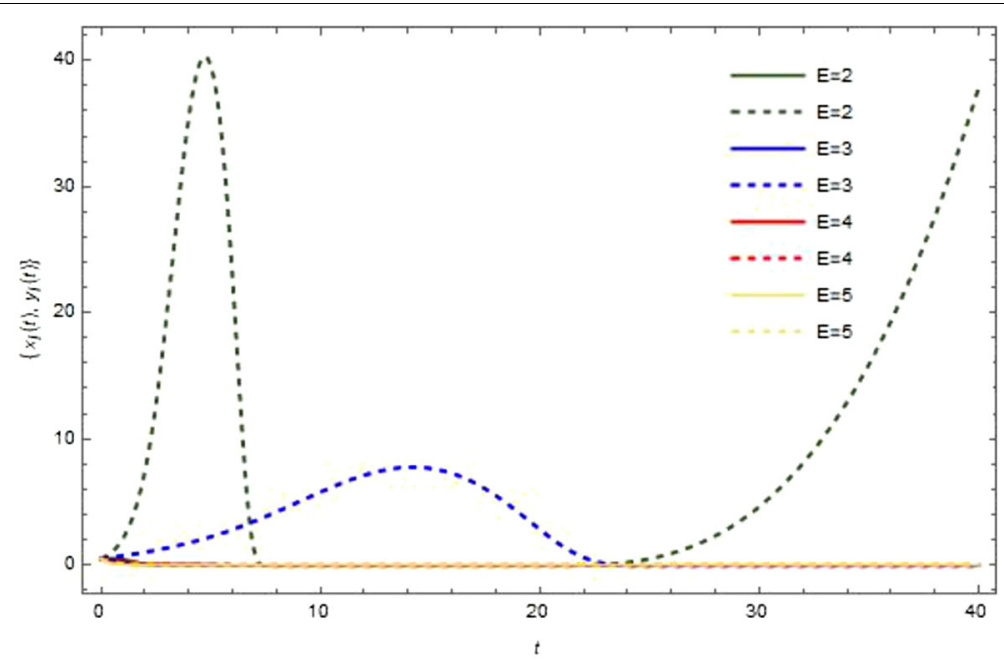

(a) $x_{l}(t ; \alpha), y_{l}(t ; \alpha)$ for $q_{1}=q_{2}=1$

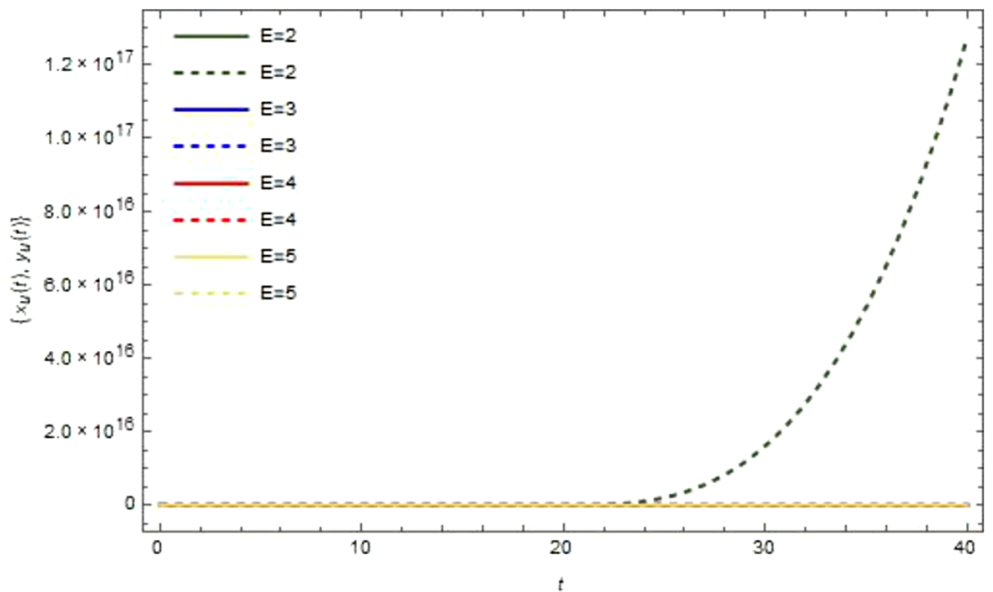

(b) $x_{u}(t ; \alpha), y_{u}(t ; \alpha)$ for $q_{1}=q_{2}=1$

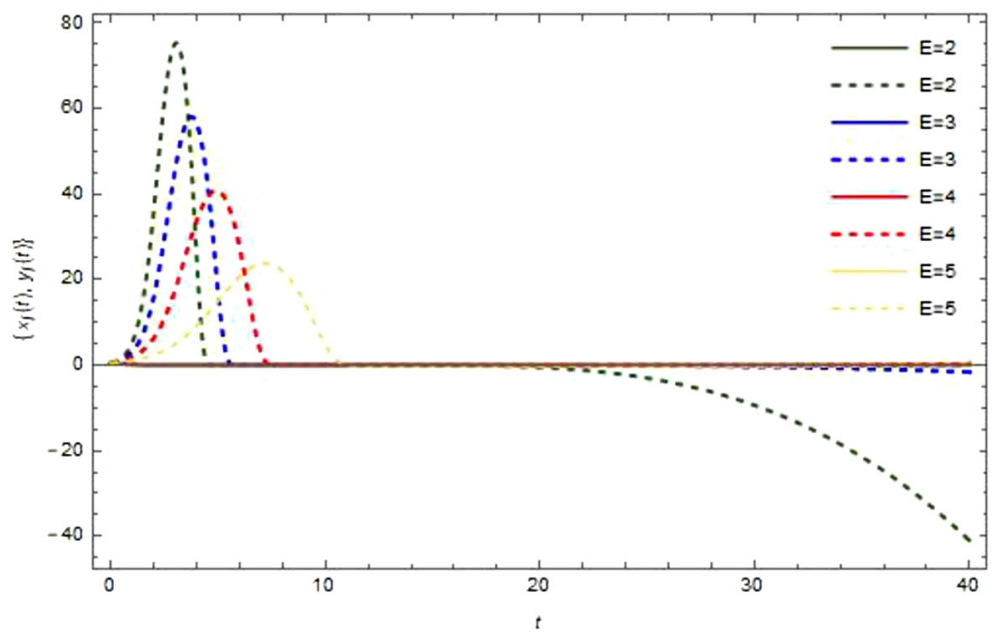

(c) $x_{l}(t ; \alpha), y_{l}(t ; \alpha)$ for $q_{1}=1, q_{2}=0.5$

Figure 2 Solutions of system (12), straight lines for $\tilde{x}(t)$ and dotted lines for $\tilde{y}(t)$, for the cases $q_{1}=q_{2}, q_{1}>q_{2}$ and $q_{1}<q_{2}$ at $\alpha=0.98, \sigma=0.998$ and for different values of $E$ 


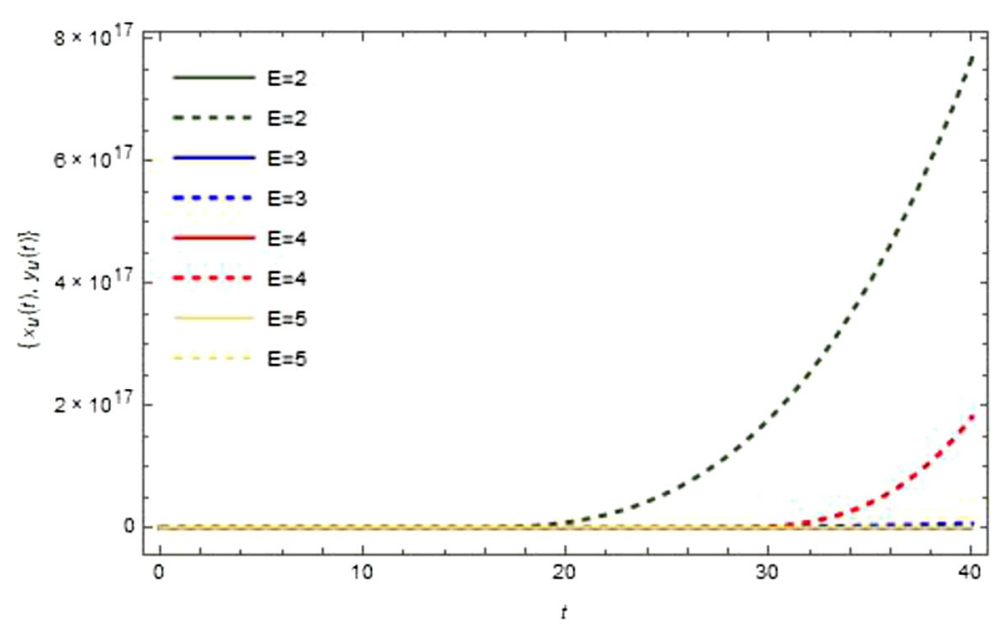

(d) $x_{u}(t ; \alpha), y_{u}(t ; \alpha)$ for $q_{1}=1, q_{2}=0.5$

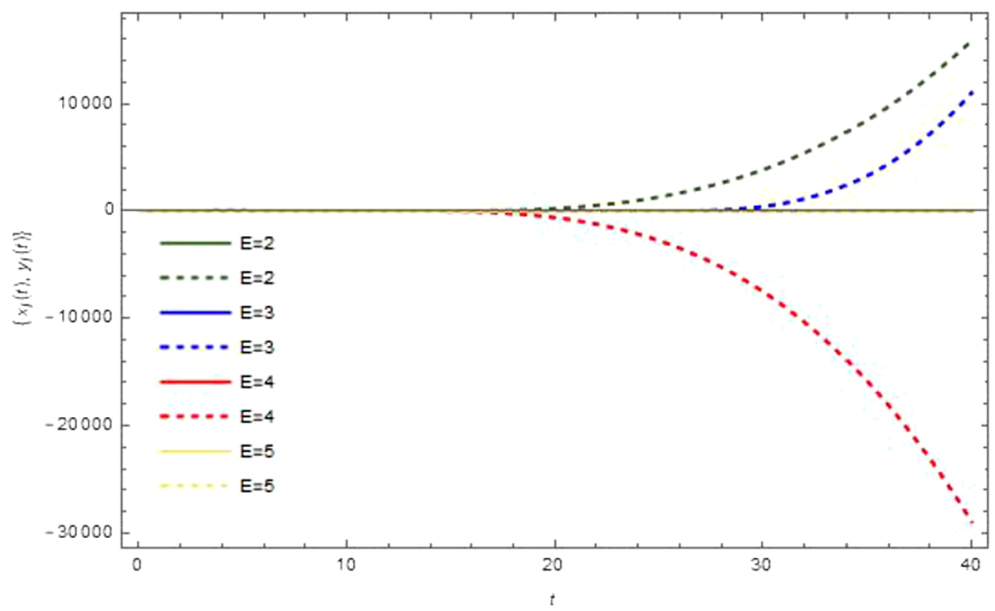

(e) $x_{l}(t ; \alpha), y_{l}(t ; \alpha)$ for $q_{1}=0.5, q_{2}=1$

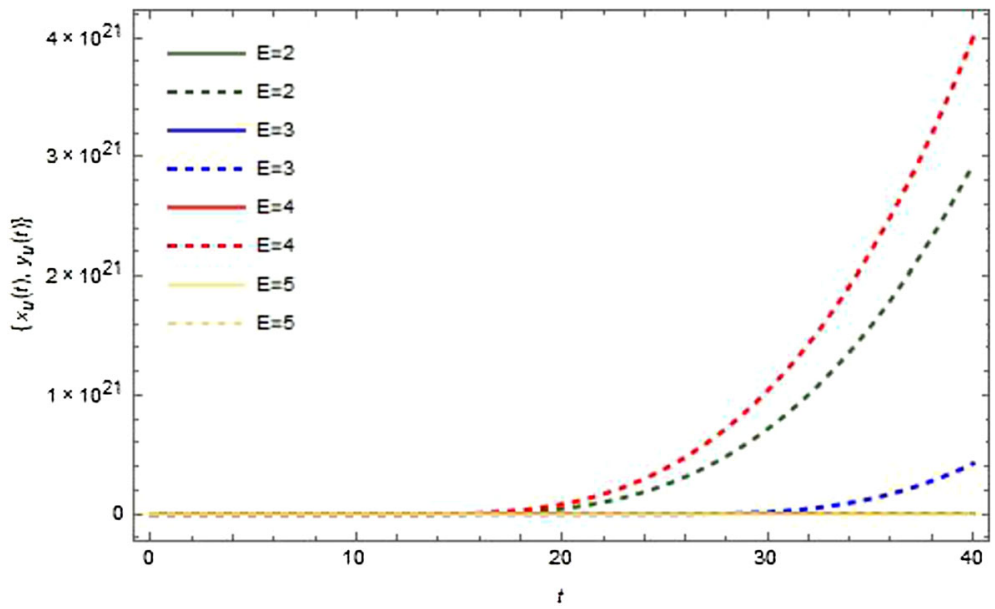

(f) $x_{u}(t ; \alpha), y_{u}(t ; \alpha)$ for $q_{1}=0.5, q_{2}=1$

Figure 2 Continued 


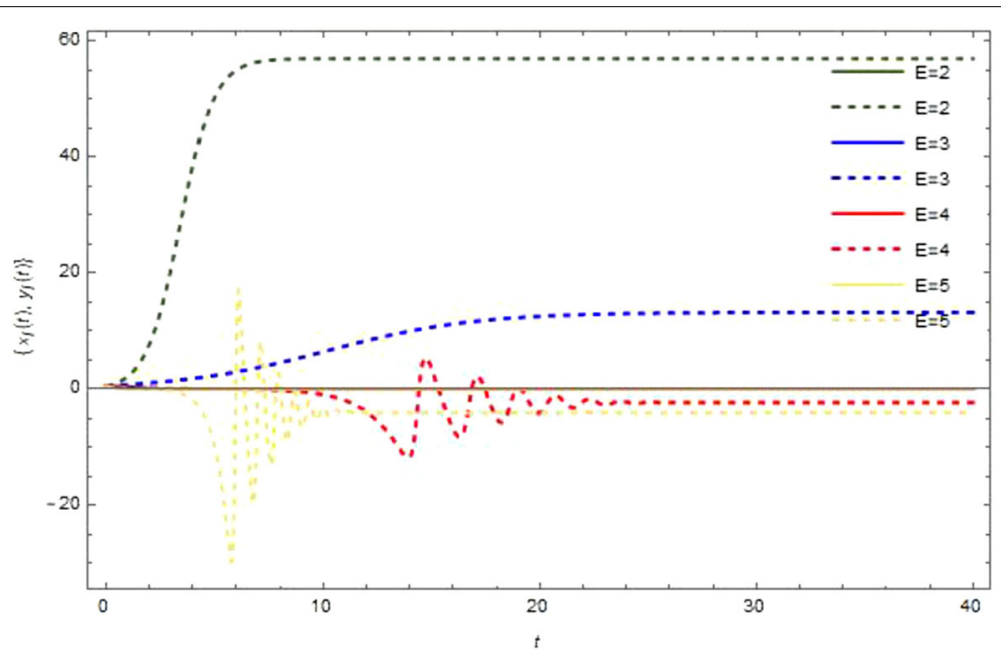

(a) $x_{l}(t ; \alpha), y_{l}(t ; \alpha)$ for $q_{1}=q_{2}=1$

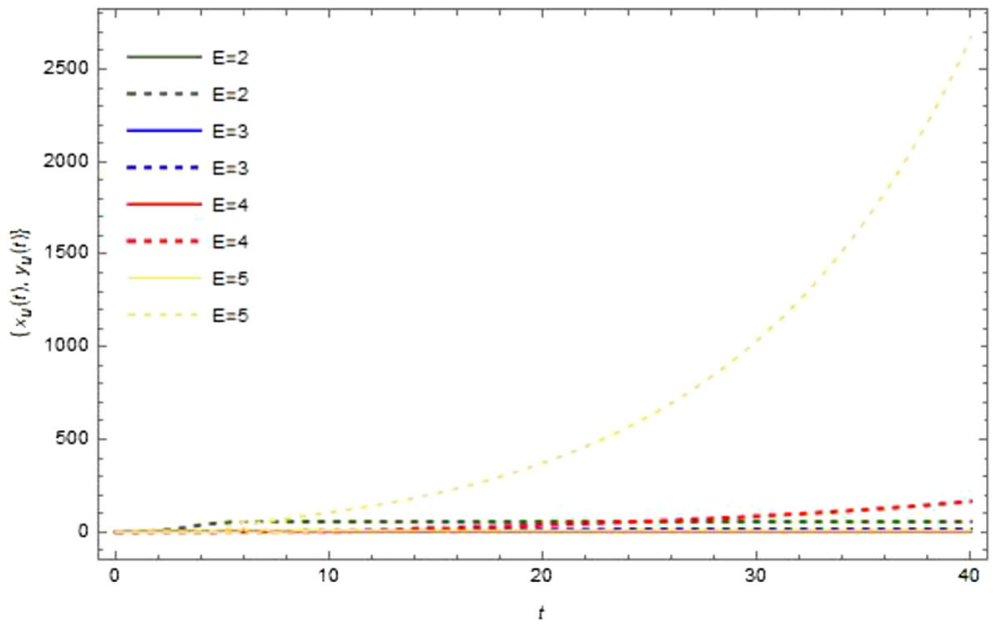

(b) $x_{u}(t ; \alpha), y_{u}(t ; \alpha)$ for $q_{1}=q_{2}=1$

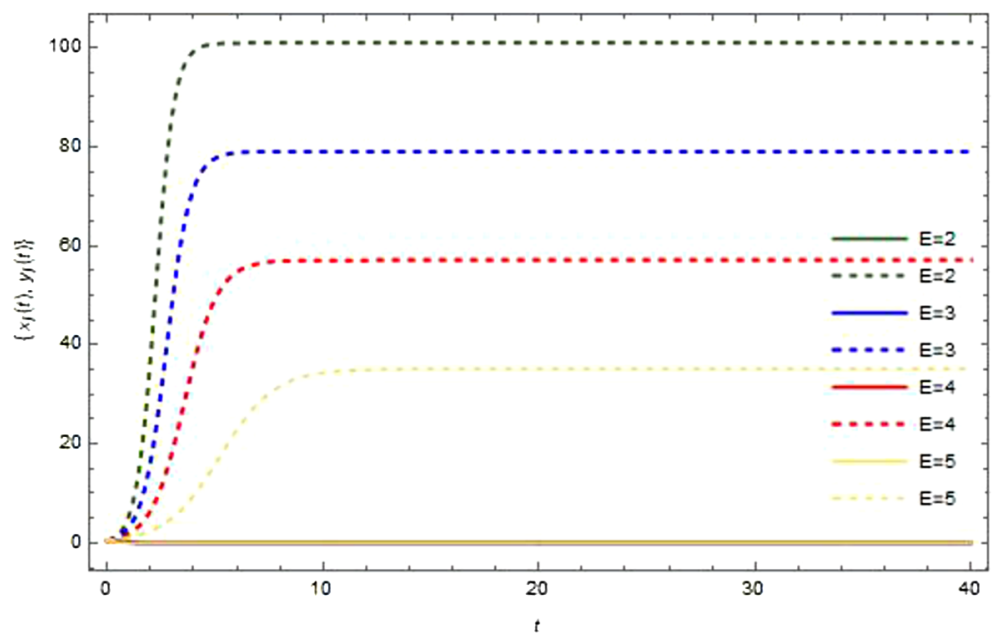

(c) $x_{l}(t ; \alpha), y_{l}(t ; \alpha)$ for $q_{1}=1, q_{2}=0.5$

Figure 3 Solutions of system (13), straight lines for $\tilde{x}(t)$ and dotted lines for $\tilde{y}(t)$, for the cases $q_{1}=q_{2}, q_{1}>q_{2}$ and $q_{1}<q_{2}$ at $\alpha=0.98, \sigma=0.998$ and for different values of $E$ 


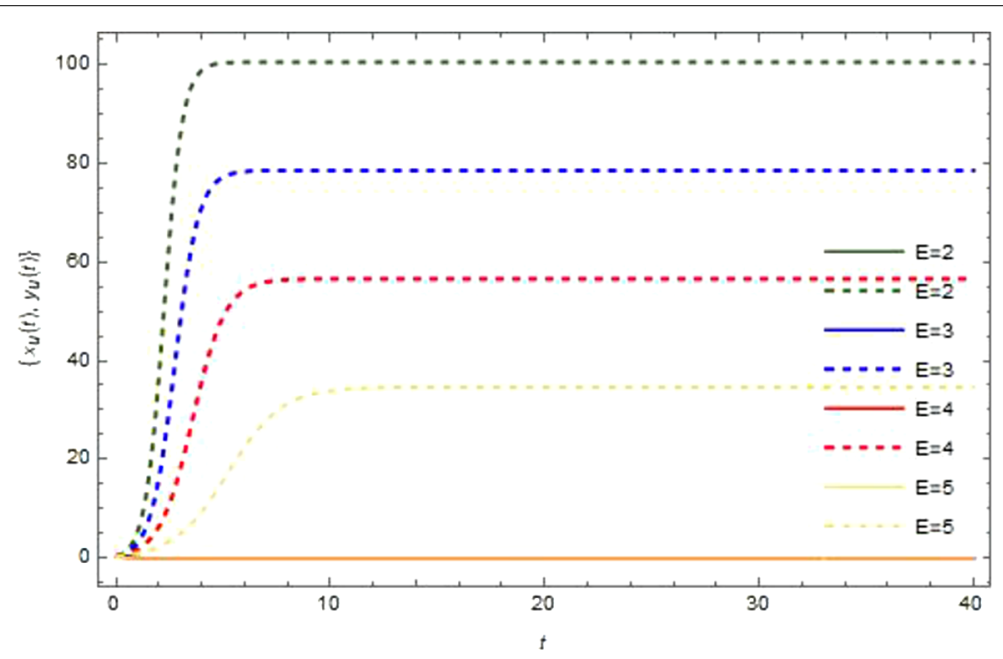

(d) $x_{u}(t ; \alpha), y_{u}(t ; \alpha)$ for $q_{1}=1, q_{2}=0.5$

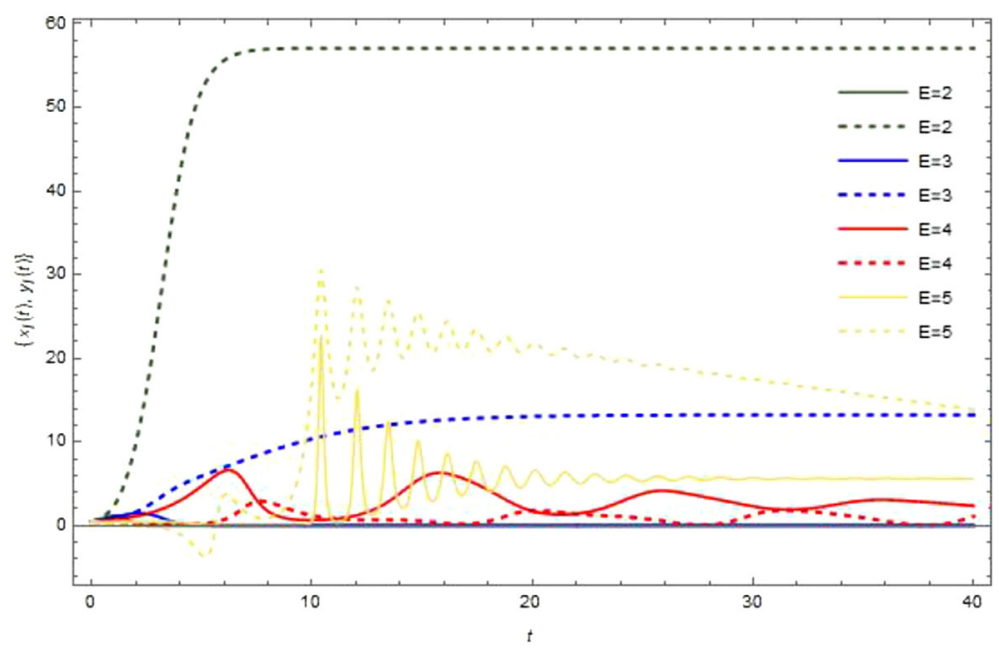

(e) $x_{l}(t ; \alpha), y_{l}(t ; \alpha)$ for $q_{1}=0.5, q_{2}=1$

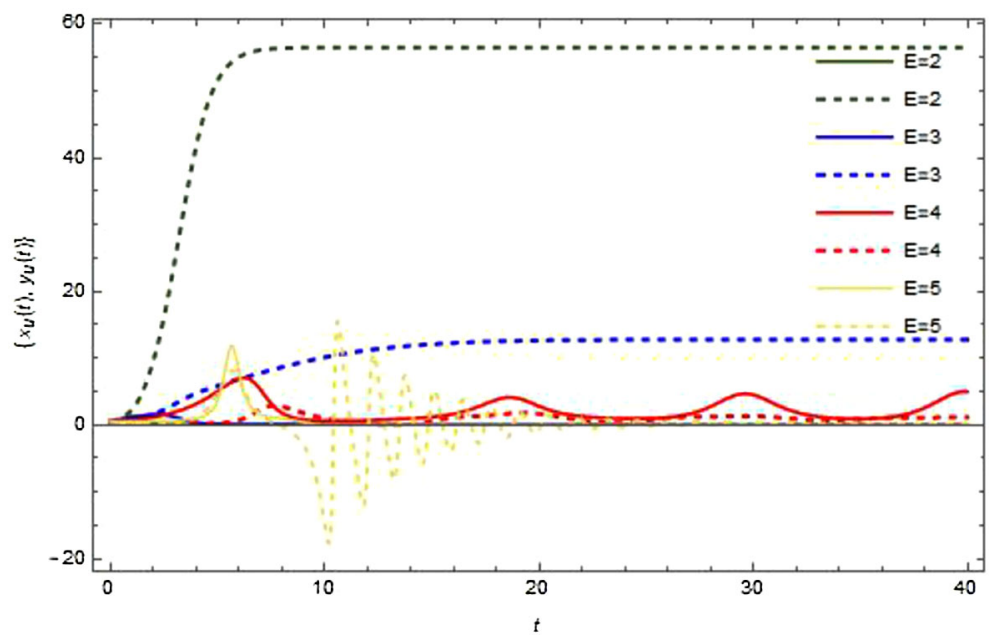

(f) $x_{u}(t ; \alpha), y_{u}(t ; \alpha)$ for $q_{1}=0.5, q_{2}=1$

Figure 3 Continued 


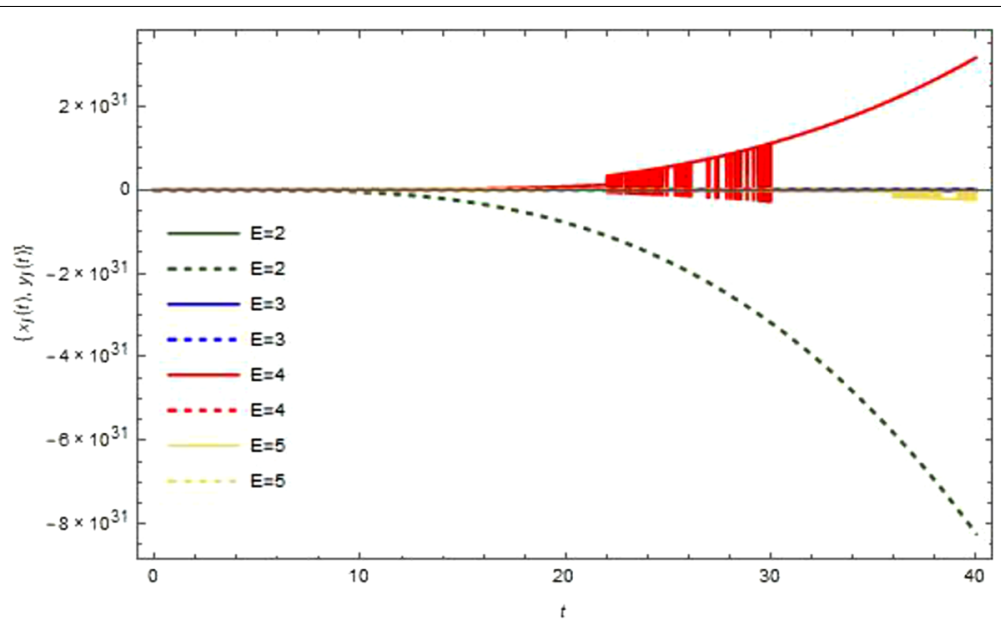

(a) $x_{l}(t ; \alpha), y_{l}(t ; \alpha)$ for $q_{1}=q_{2}=1$

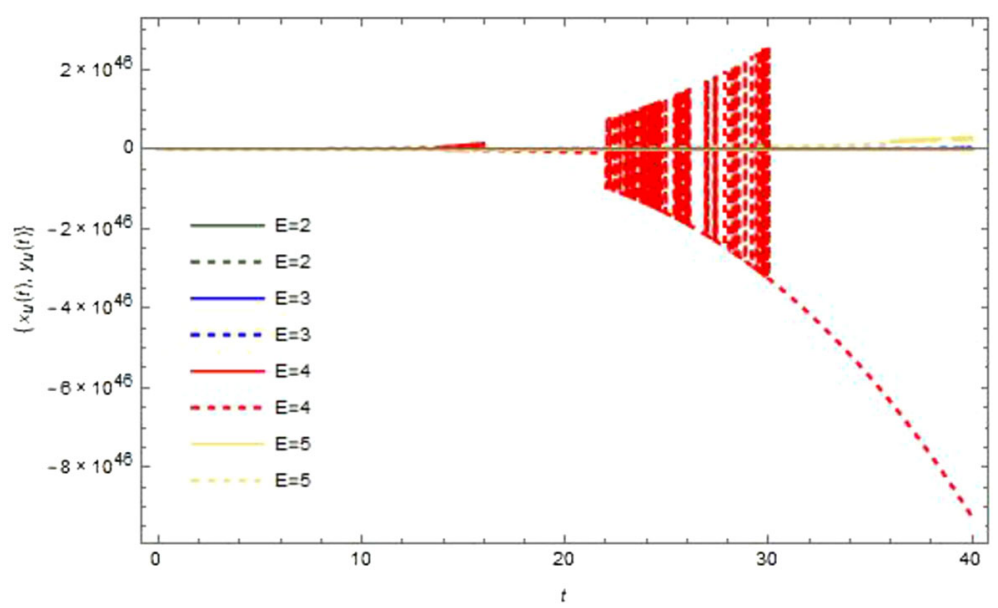

(b) $x_{u}(t ; \alpha), y_{u}(t ; \alpha)$ for $q_{1}=q_{2}=1$.

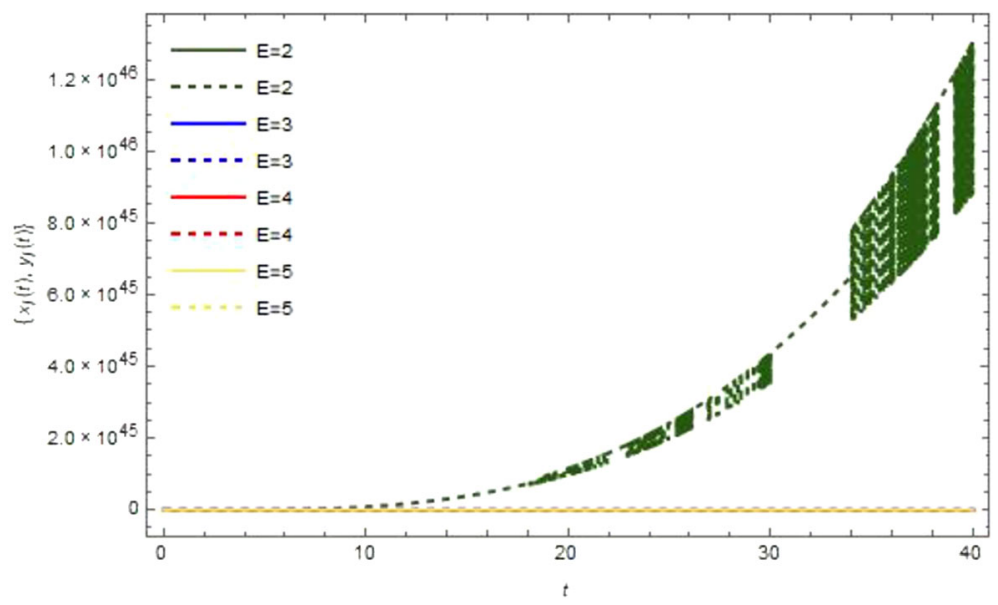

(c) $x_{l}(t ; \alpha), y_{l}(t ; \alpha)$ for $q_{1}=1, q_{2}=0.5$

Figure 4 Solutions of system (14), straight lines for $\tilde{x}(t)$ and dotted lines for $\tilde{y}(t)$, for the cases $q_{1}=q_{2}, q_{1}>q_{2}$ and $q_{1}<q_{2}$ at $\alpha=0.98, \sigma=0.998$ and for different values of $E$ 


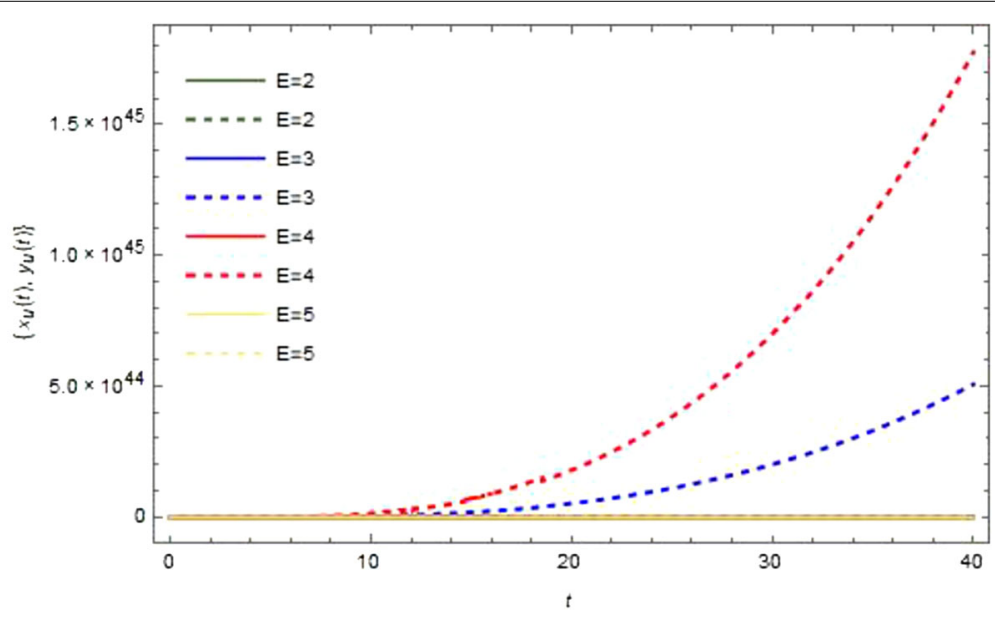

(d) $x_{u}(t ; \alpha), y_{u}(t ; \alpha)$ for $q_{1}=1, q_{2}=0.5$

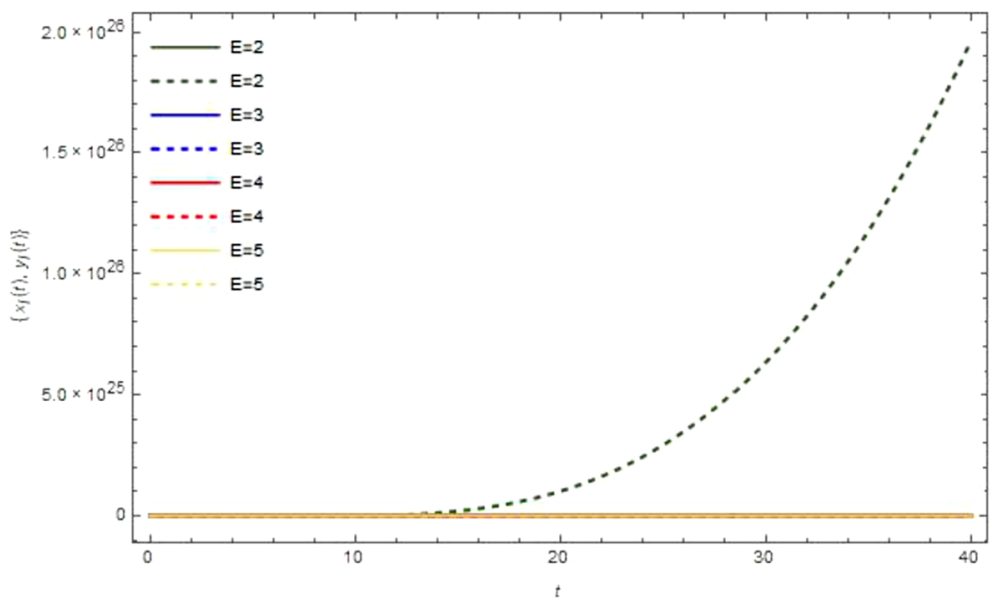

(e) $x_{l}(t ; \alpha), y_{l}(t ; \alpha)$ for $q_{1}=0.5, q_{2}=1$

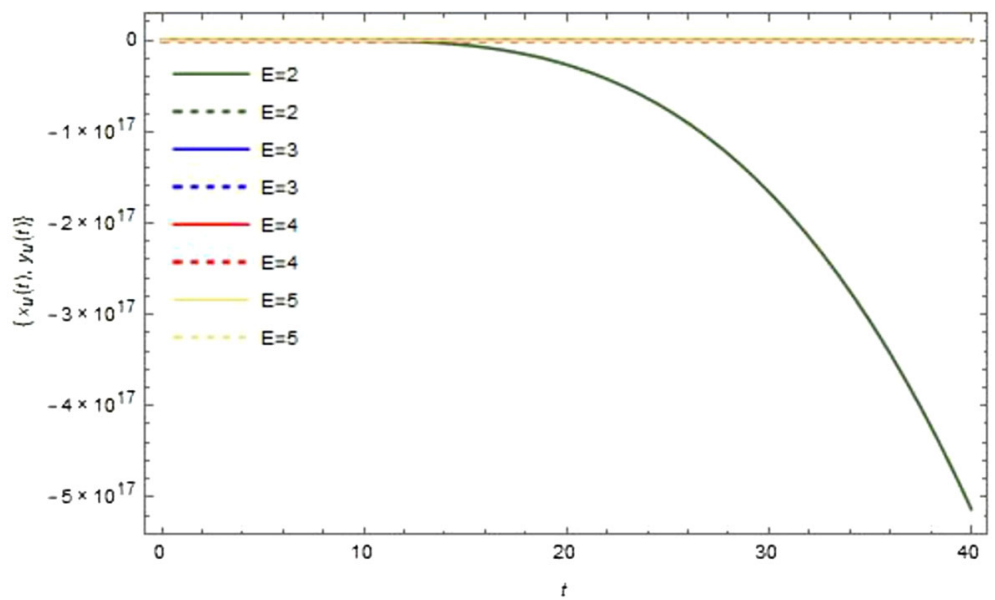

(f) $x_{u}(t ; \alpha), y_{u}(t ; \alpha)$ for $q_{1}=0.5, q_{2}=1$.

Figure 4 Continued 


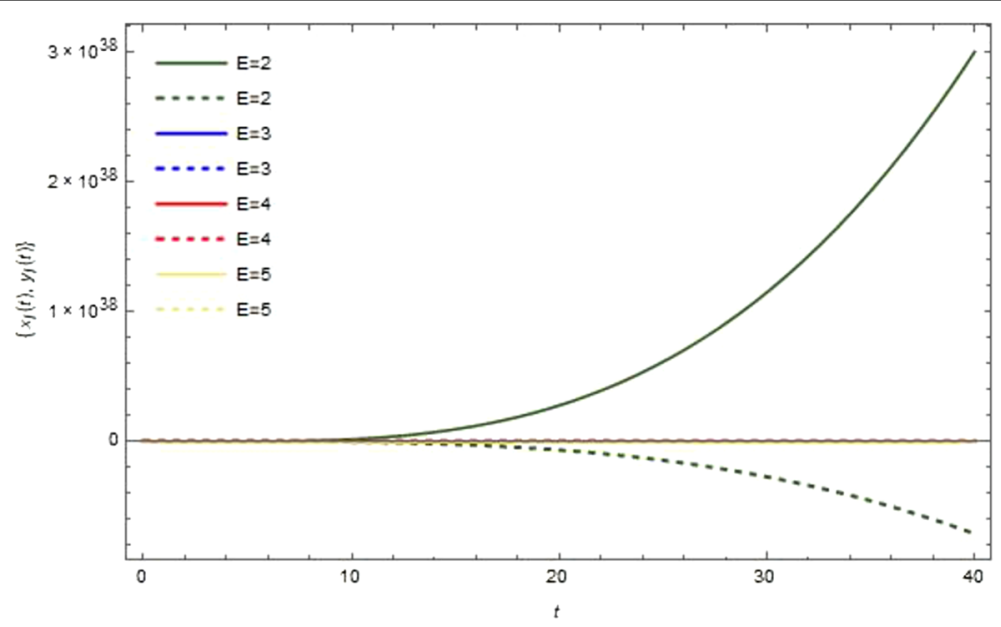

(a) $x_{l}(t ; \alpha), y_{l}(t ; \alpha)$ for $q_{1}=q_{2}=1$

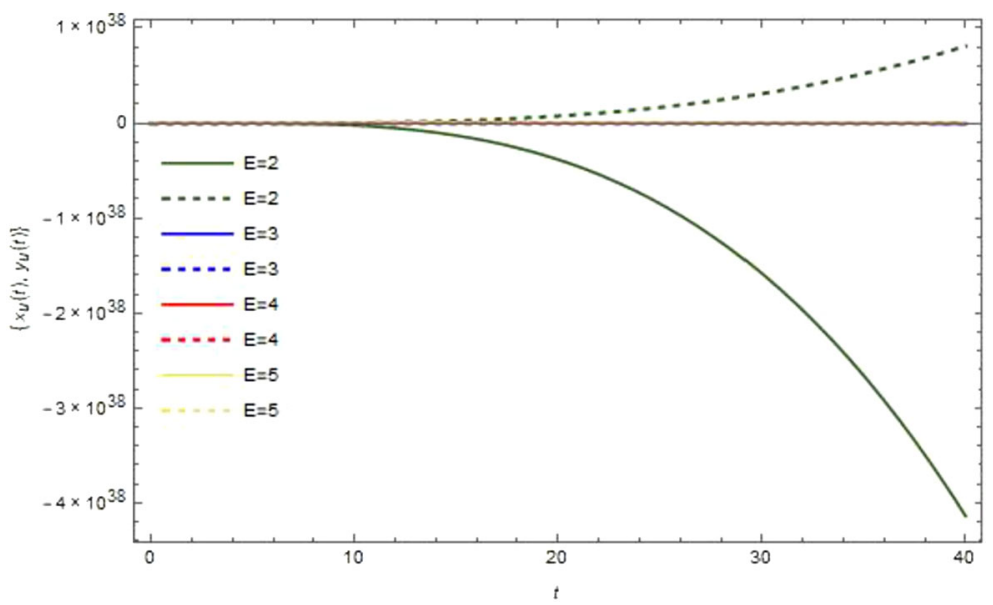

(b) $x_{u}(t ; \alpha), y_{u}(t ; \alpha)$ for $q_{1}=q_{2}=1$

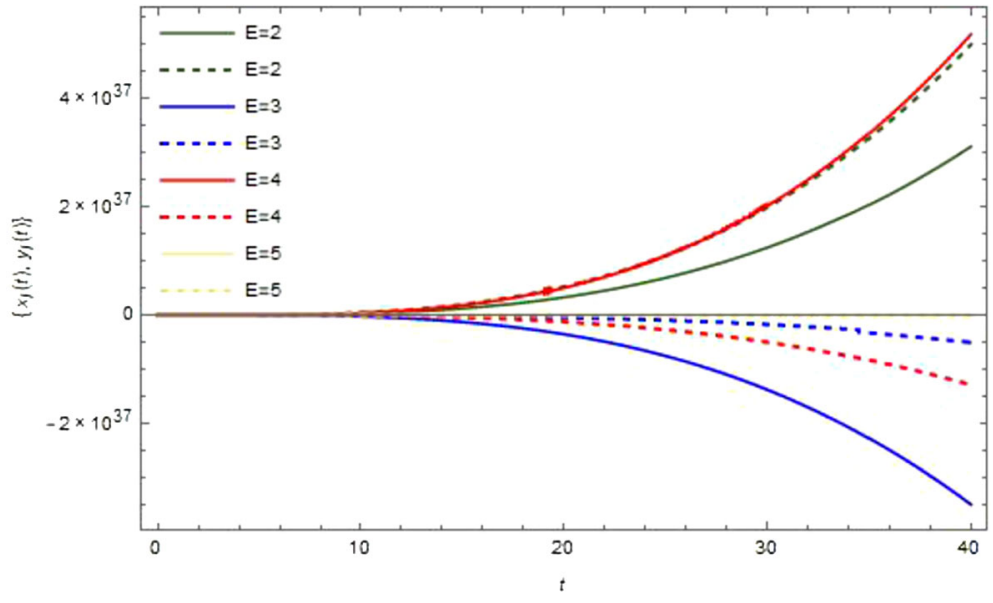

(c) $x_{l}(t ; \alpha), y_{l}(t ; \alpha)$ for $q_{1}=1, q_{2}=0.5$

Figure 5 Solutions of system (15), straight lines for $\tilde{x}(t)$ and dotted lines for $\tilde{y}(t)$, for the cases $q_{1}=q_{2}, q_{1}>q_{2}$ and $q_{1}<q_{2}$ at $\alpha=0.98, \sigma=0.998$ and for different values of $E$ 


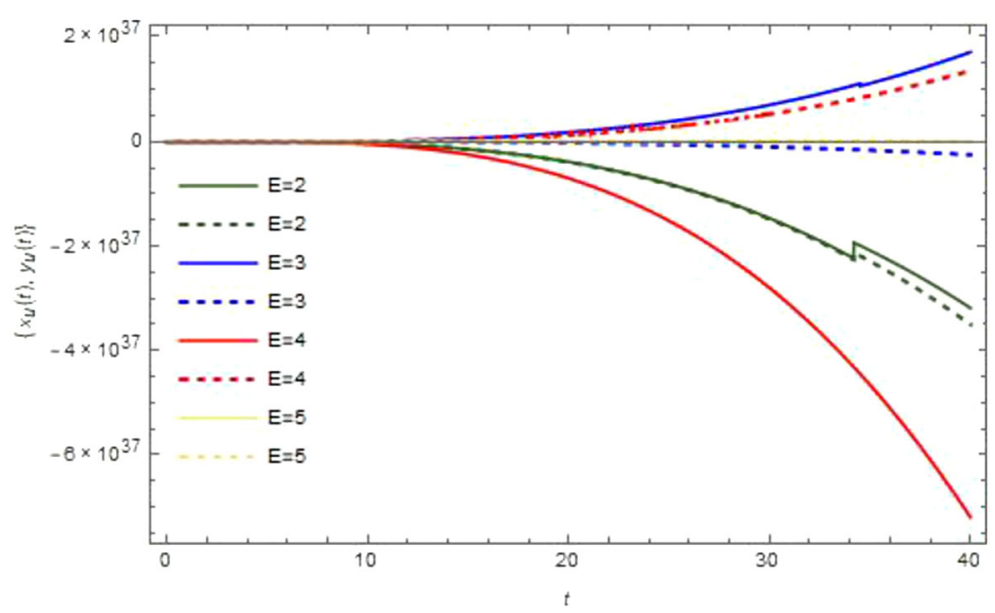

(d) $x_{u}(t ; \alpha), y_{u}(t ; \alpha)$ for $q_{1}=1, q_{2}=0.5$

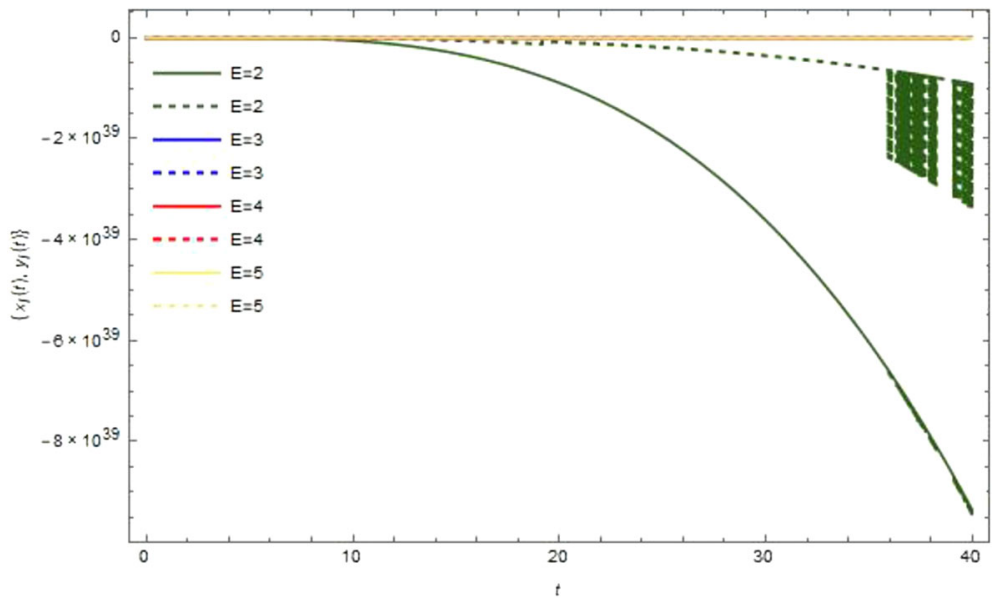

(e) $x_{l}(t ; \alpha), y_{l}(t ; \alpha)$ for $q_{1}=0.5, q_{2}=1$

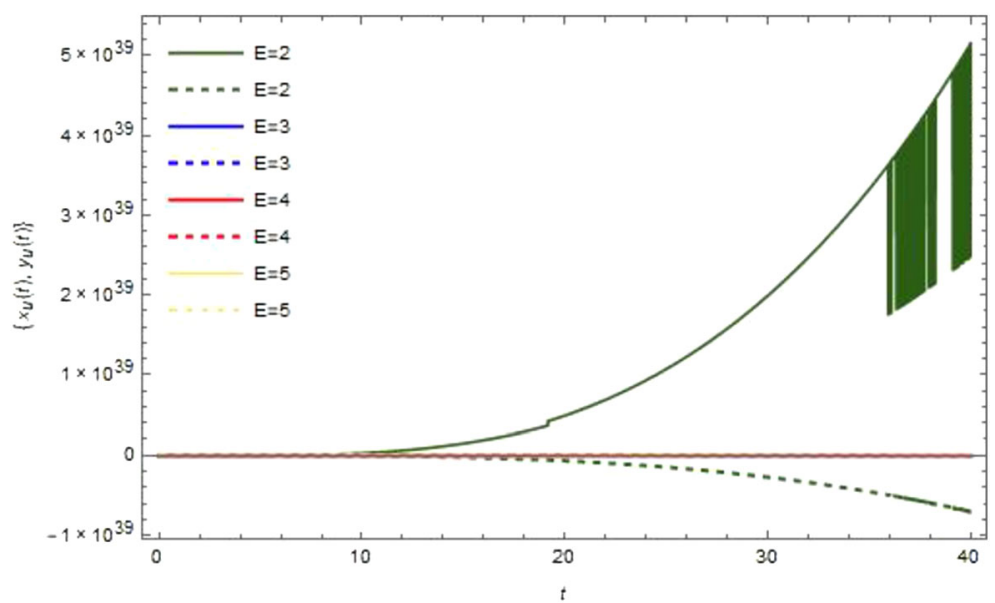

(f) $x_{u}(t ; \alpha), y_{u}(t ; \alpha)$ for $q_{1}=0.5, q_{2}=1$

Figure 5 Continued 


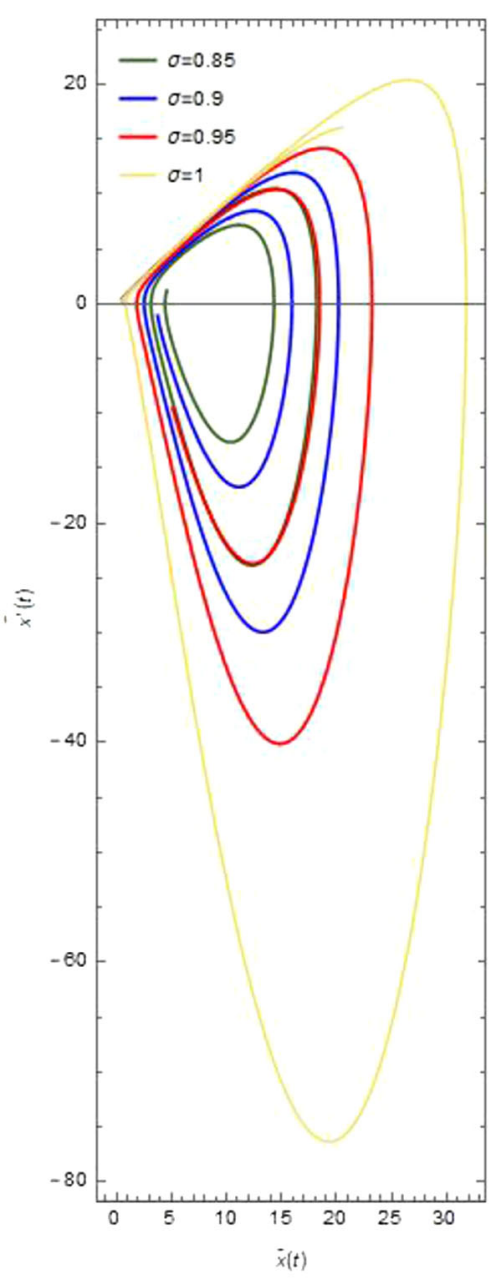

(a) Planes of $\tilde{x}(t)$ for system (12)

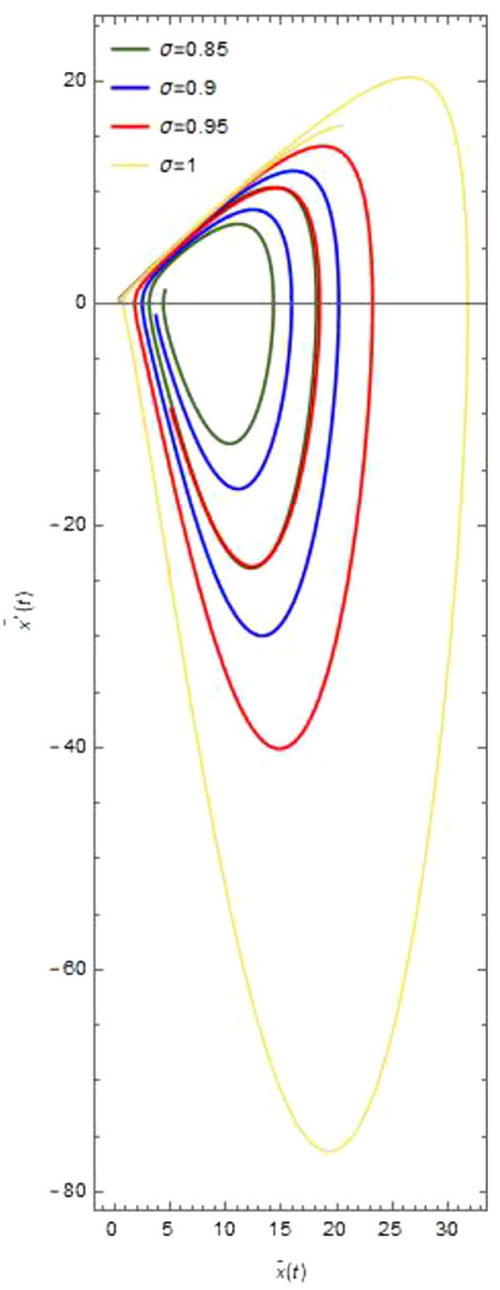

(b) Planes of $\tilde{x}(t)$ for system (13)

Figure 6 Phase planes for $\alpha=1, q_{1}=0.2, q_{2}=0.7, t \in[0,10]$ and $E=8$

\section{Conclusions}

In this paper, an ecological model was discussed in fuzzy environment with fractional order derivative by using fuzzy fractional Hukuhara differentiability approach. We described the two interacting species model with the stability of its equilibrium points by using the variational matrix Routh-Hurwitz condition. Fuzzy solutions of different fgHdifferentiability cases of fuzzy functions were discussed. Moreover, some theorems were presented that provide different conditions for harvesting of the species, which would lead to the stability of the population of prey and predator. To support the demonstrations, graphical and numerical dynamics of prey and predator were represented for the corresponding systems with the help of fuzzy fractional Laplace transform expansion and FFRK, GL, and ABM. Consequently, we came up with the following outcomes:

- The imprecision of the environment may cause complexities while studying the interaction of species.

- Fractional order derivative intriguingly made it easy to locate the historical position and patterns of each limit cycle of the governing systems. 


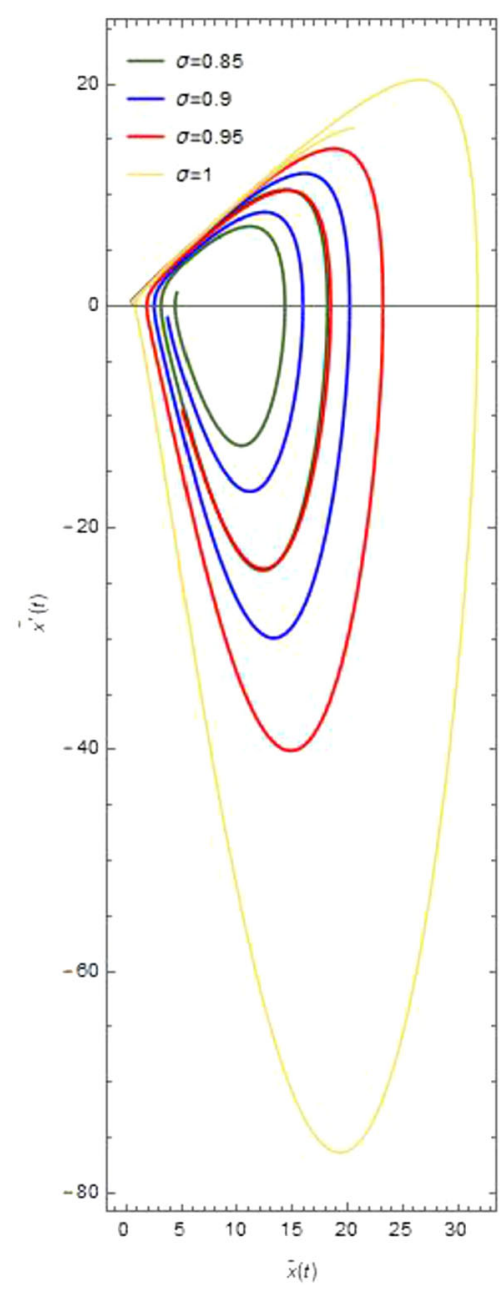

(c) Planes of $\tilde{x}(t)$ for system (14)

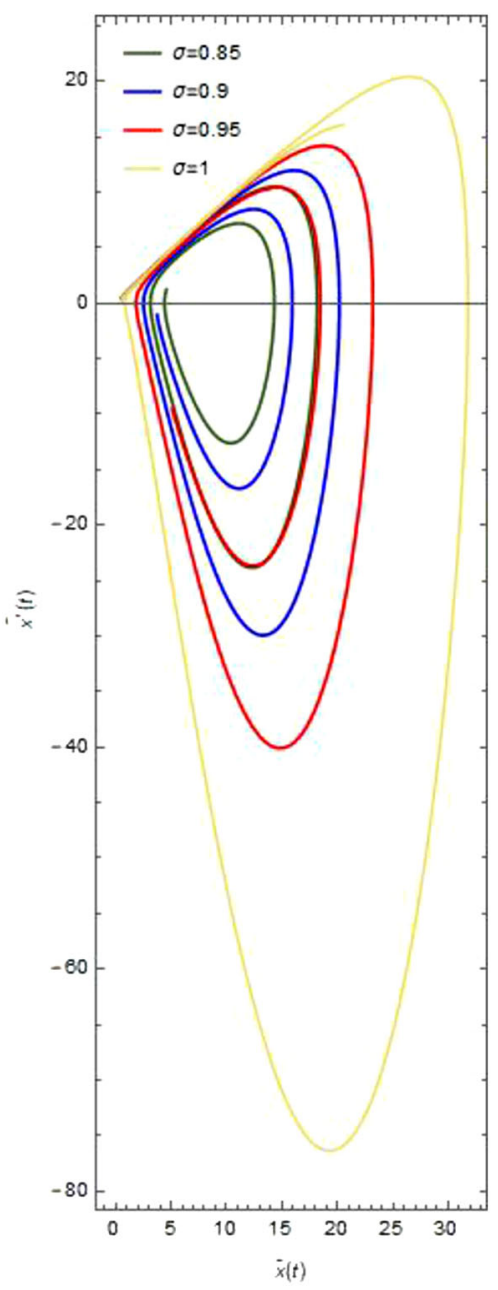

(d) Planes of $\tilde{x}(t)$ for system (15)

Figure 6 Continued

- The population of both the species, $\tilde{x}(t)$ and $\tilde{y}(t)$, goes to extinction if the harvesting rate $E$ is greater than the ratio of logistic growth and catchability coefficients.

- If the harvesting rate $E$ of both the species is less than the ratio of imprecise biological parameters, $\tilde{r}_{1}, \tilde{r}_{2}, \tilde{b}_{11}, \tilde{b}_{12}, \tilde{b}_{21}, \tilde{b}_{22}, \tilde{k}_{1}$, and $\tilde{k}_{2}$ increase, as $\alpha$ increases, the population of both the species, $\tilde{x}(t)$ and $\tilde{y}(t)$, becomes stable.

- The phase plane trajectories show a gradual increase in the coexistence intervals of prey and predator, as the fractional variation in time moves toward the integer order.

Hence, it is concluded that the interaction of the species depends on the imprecise nature of the ecological parameters, mainly the harvesting threshold, when both species undergo economical hunting. Accordingly, the approach is very helpful for the researchers who are involved in mathematical modeling with imprecision in various fields of science and engineering. It reveals very realistic results in both mathematical and ecological point of view. There is still a room for further research in this field, thus, many breakthrough studies can be explored with fuzzy parameters and fractional order derivative. 


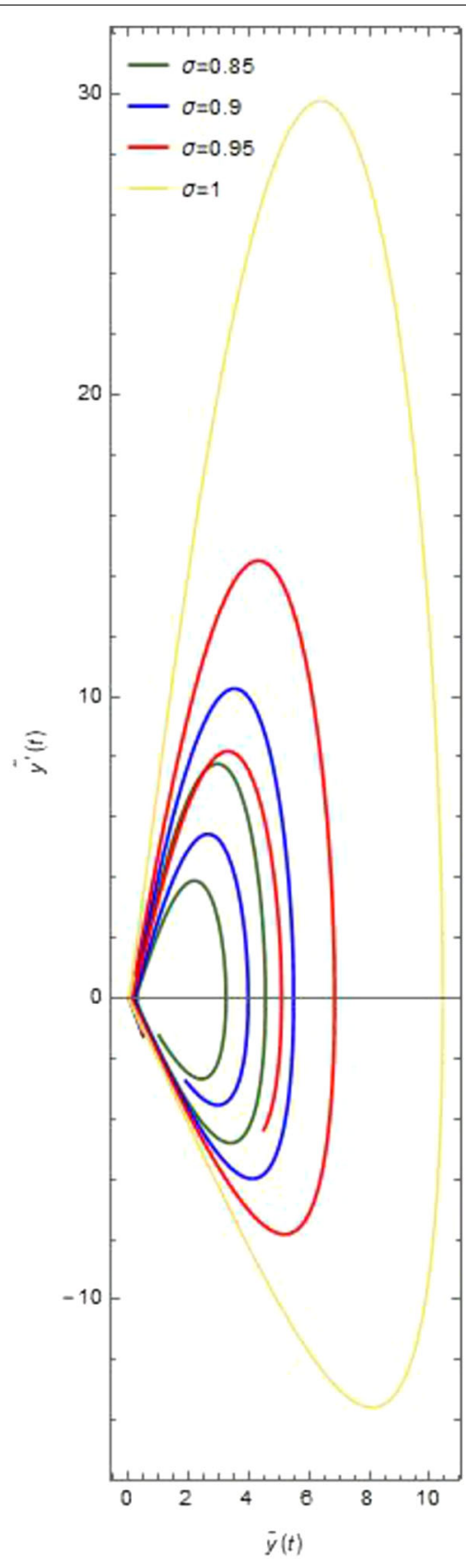

(a) Planes of $\tilde{y}(t)$ for system (12)

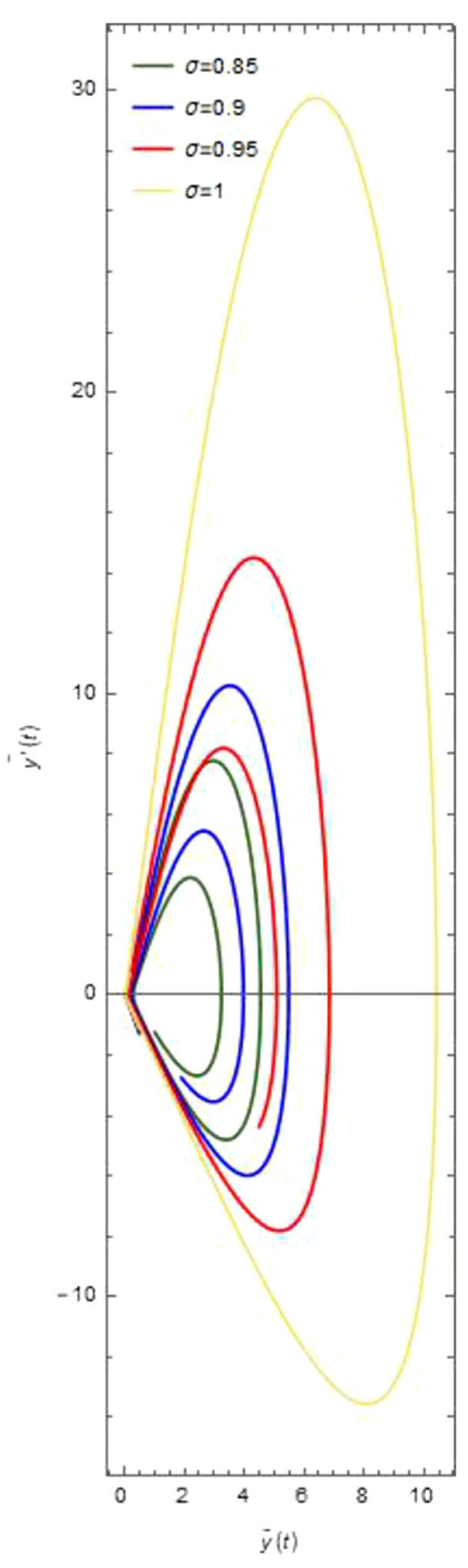

(b) Planes of $\tilde{y}(t)$ for system (13)

Figure 7 Phase planes for $\alpha=1, q_{1}=0.2, q_{2}=0.7, t \in[0,10]$ and $E=8$ 


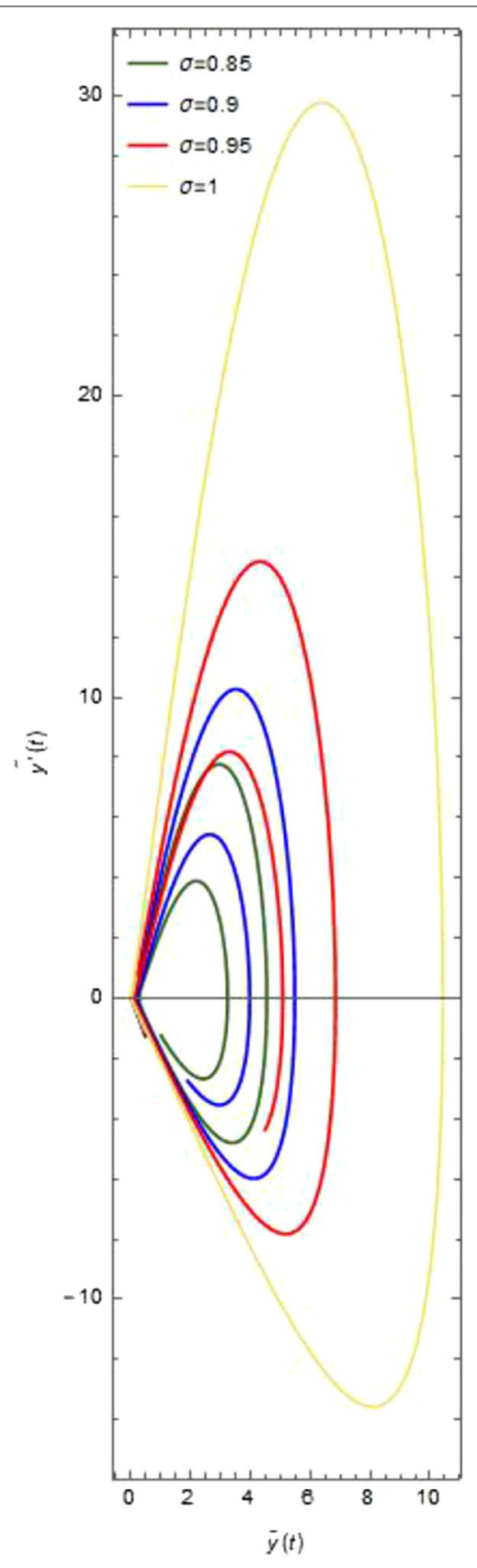

(c) Planes of $\tilde{y}(t)$ for system (14)

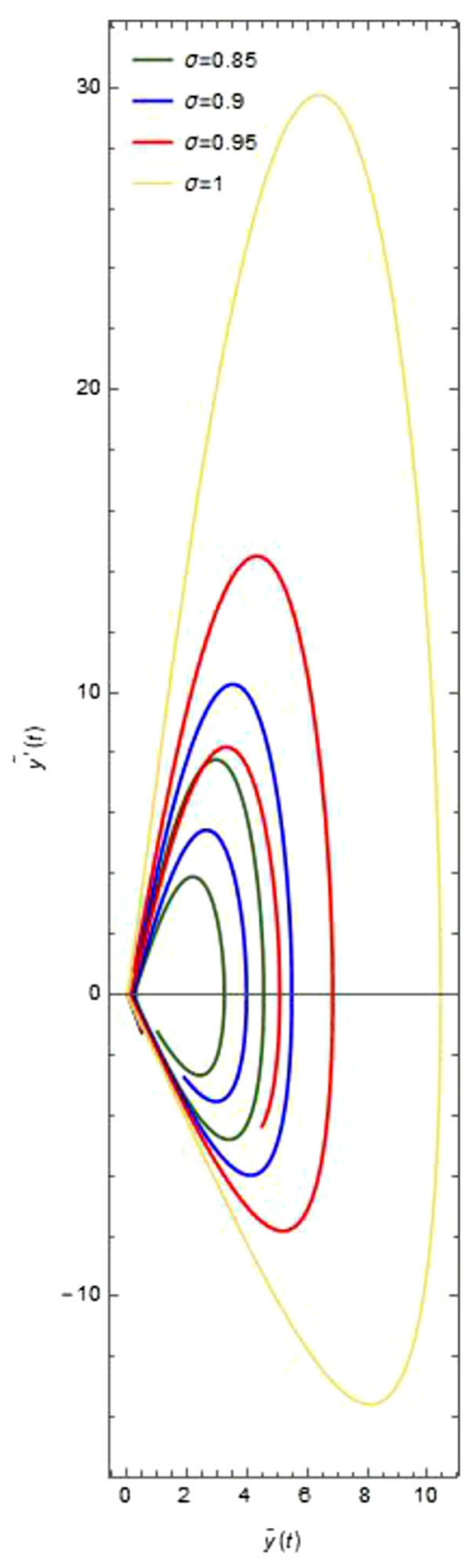

(d) Planes of $\tilde{y}(t)$ for system (15)

Figure 7 Continued 


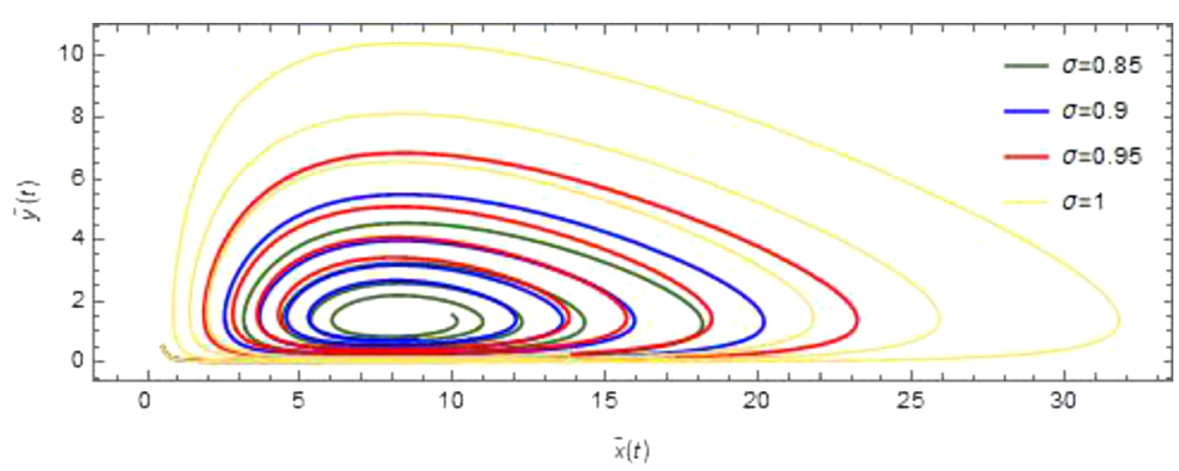

(a) Trajectories of system (12) for different values of $\sigma$

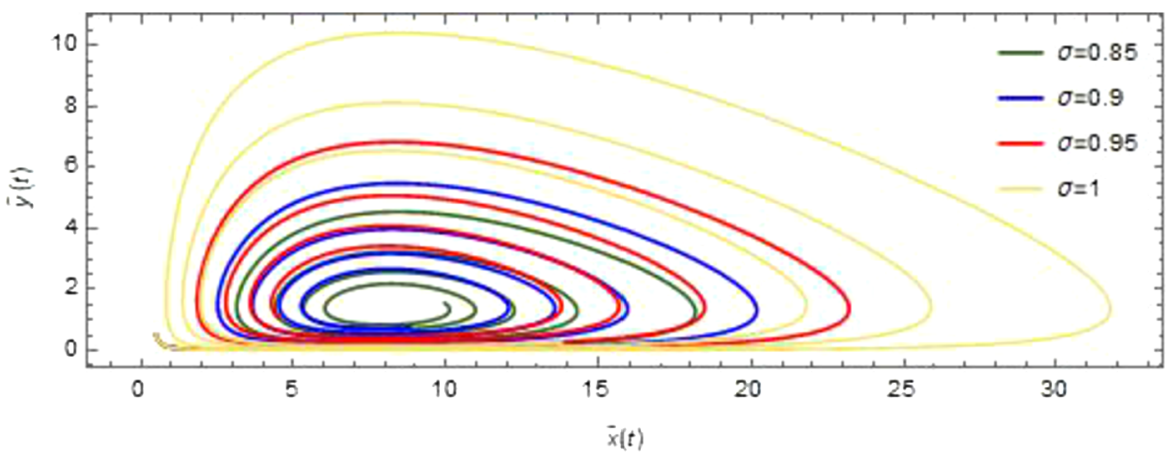

(b) Trajectories of system (13) for different values of $\sigma$

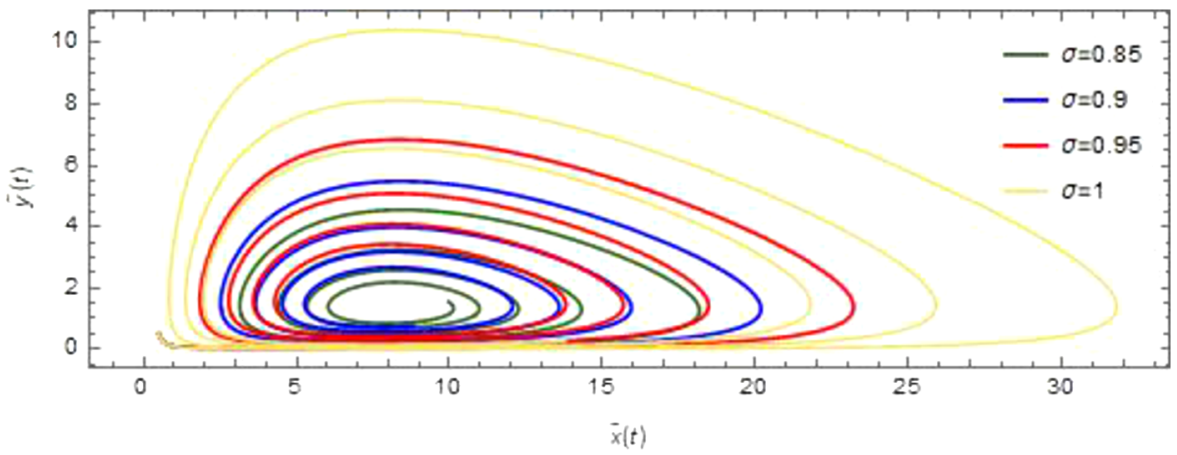

(c) Trajectories of system (14) for different values of $\sigma$

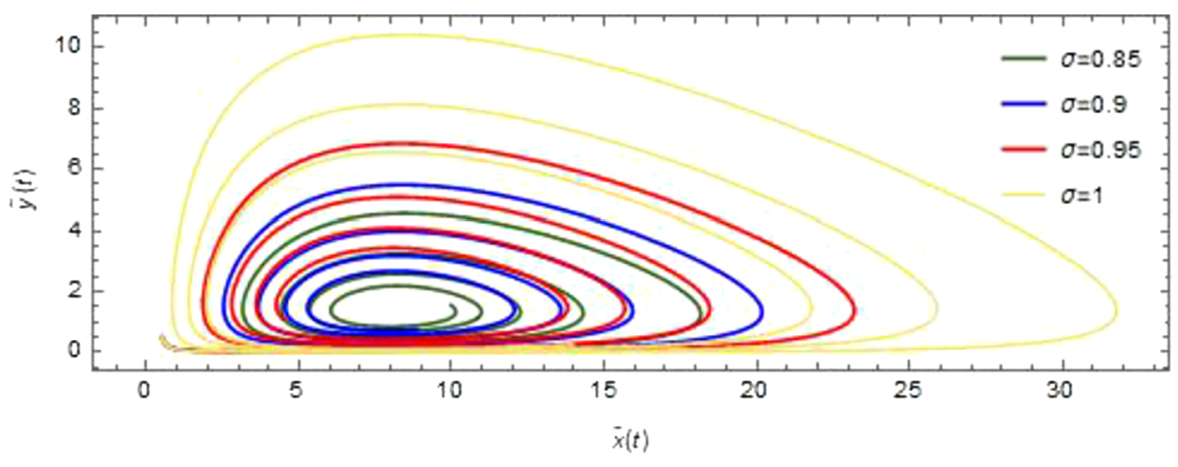

(d) Trajectories of system (15) for different values of $\sigma$

Figure 8 Limit cycles of the prey-predator model for $\alpha=1, q_{1}=0.2, q_{2}=0.7, t \in[0,20]$ and $E=8$ 


\section{Acknowledgements}

The author OAR is thankful to Bahria University for supporting/facilitating this work. All the authors are thankful to both referees for fruitful comments.

\section{Funding}

There is no research funding.

\section{Competing interests}

The authors declare that they have no conflict of interest

\section{Research involving human participants and/or animals:}

No research/data involve human participants and/or animals.

\section{Authors' contributions}

All authors contributed equally to this work. All authors read and approved the final manuscript.

\section{Author details}

'Department of Mathematics, University of Karachi, Karachi, Pakistan. ${ }^{2}$ Department of Humanities \& Social Sciences, Bahria University, Karachi, Pakistan. ${ }^{3}$ Department of Applied Science, Maulana Abul Kalam Azad University of Technology, West Bengal, India. ${ }^{4}$ Department of Mathematics, The Women University Multan, Multan, Pakistan.

\section{Publisher's Note}

Springer Nature remains neutral with regard to jurisdictional claims in published maps and institutional affiliations.

Received: 13 December 2018 Accepted: 5 September 2019 Published online: 23 September 2019

\section{References}

1. Lotka, A.J.: Elements of physical biology. Sci. Prog. Twent. Cent. (1919-1933) 21(82), 341-343 (1926)

2. Volterra, V.: Variazioni efluttuazioni del numero di individui in specie animali conviventi. In: Memoria della Reale Accademia Nazionale dei Lince pp. 31-113 (1927)

3. Chakraborty, B., Bairagi, N.: Complexity in a prey-predator model with prey refuge and diffusion. Ecol. Complex. 37, $11-23(2019)$

4. Mukhopadhyay, B., Bhattacharyya, R.: Effects of harvesting and predator interference in a model of two-predators competing for a single prey. Appl. Math. Model. 40(4), 3264-3274 (2016)

5. Wu, C.C.: The spreading speed for a predator-prey model with one predator and two preys. Appl. Math. Lett. 91, 9-14 (2019)

6. Das, A., Samanta, G.P.: Stochastic prey-predator model with additional food for predator. Phys. A, Stat. Mech. Appl. 512,121-141 (2018)

7. Pal, D., Mahapatra, G.S.: A bioeconomic modeling of two-prey and one-predator fishery model with optimal harvesting policy through hybridization approach. Appl. Math. Comput. 242, 748-763 (2014)

8. Nounou, H.N., Nounou, M.N., Meskin, N., Datta, A., Dougherty, E.R.: Fuzzy intervention in biological phenomena. IEEE/ACM Trans. Comput. Biol. Bioinform. 9(6), 1819-1825 (2012)

9. Paul, S., Mondal, S.P., Bhattacharya, P.: Discussion on fuzzy quota harvesting model in fuzzy environment: fuzzy differential approach. Model. Earth Syst. Environ. 2, 70 (2016). https://doi.org/10.1007/s40808-016-0113-y

10. Paul, S., Mondal, S.P., Bhattacharya, P.: Numerical solution of Lotka-Volterra prey predator model by using Runge-Kutta-Fehlberg method and Laplace-Adomian decomposition method. Alex. Eng. J. 55(1), 613-617 (2016)

11. Barzinji, K., Maan, N.: Fuzzy delay predator-prey system: existence theorem and oscillation property of solution. Int. J. Math. Anal. 8(17), 829-847 (2014)

12. Pandit, P., Singh, P.: Prey predator model with fuzzy initial conditions. Int. J. Eng. Innov. Technol. 3(12), 65-68 (2014)

13. Tapaswini, S., Chakraverty, S.: Numerical solution of fuzzy arbitrary order predator-prey equations. Appl. Appl. Math. 8(2), 647-672 (2013)

14. Arumugam, D., Uduman, P.S.S.: Fuzzy fractional order predator-prey model for predicting the time of hypertension in pregnancy. Int. J. Pure Appl. Math. 109(5), 75-83 (2016)

15. Khan, N.A., Razzaq, O.A., Ara, A., Riaz, F.: Numerical solution of system of fractional differential equations in imprecise environment. In: Num. Simul. Brain Imag. Turb. Flow, pp. 167-186. IntechOpen, London (2016)

16. Kumar, D., Rai, K.N.: Numerical simulation of time fractional dual-phase-lag model of heat transfer within skin tissue during thermal therapy. J. Therm. Biol. 67, 49-58 (2017)

17. Meng, R., Yin, D., Drapaca, C.S.: Variable-order fractional description of compression deformation of amorphous glassy polymers. Comput. Mech. (2019). https://doi.org/10.1007/s00466-018-1663-9

18. Singh, J: A new analysis for fractional rumor spreading dynamical model in a social network with Mittag-Leffler law. Chaos 29(1), 013137 (2019)

19. Singh, J., Kumar, D., Baleanu, D.: On the analysis of fractional diabetes model with exponential law. Adv. Differ. Equ. 2018, 231 (2018). https://doi.org/10.1186/s13662-018-1680-1

20. Singh, J., Kumar, D., Baleanu, D., Rathore, S.: An efficient numerical algorithm for the fractional Drinfeld-Sokolov-Wilson equation. Appl. Math. Comput. 335, 12-24 (2018)

21. Singh, J., Kumar, D., Hammouch, Z., Atangana, A.: A fractional epidemiological model for computer viruses pertaining to a new fractional derivative. Appl. Math. Comput. 316, 504-515 (2018)

22. Singh, J., Kumar, D., Baleanu, D.: New aspects of fractional Biswas-Milovic model with Mittag-Leffler law. Math. Model. Nat. Phenom. 14(3), 303 (2019)

23. Kumar, D., Singh, J., Baleanu, D., Rathore, S.: Analysis of regularized long-wave equation associated with a new fractional operator with Mittag-Leffler type kernel. Phys. A, Stat. Mech. Appl. 492, 155-167 (2018) 
24. Hajipour, M., Jajarmi, A., Baleanu, D., Sun, H.G.: On an accurate discretization of a variable-order fractional reaction-diffusion equation. Commun. Nonlinear Sci. Numer. Simul. 69, 119-133 (2019)

25. Baleanu, D., Jajarmi, A., Hajipour, M.: On the nonlinear dynamical systems within the generalized fractional derivatives with Mittag-Leffler kernel. Nonlinear Dyn. 94(1), 397-414 (2018)

26. Jajarmi, A., Baleanu, D.: A new fractional analysis on the interaction of HIV with CD4+ T-cells. Chaos Solitons Fractals $113,221-229(2018)$

27. Baleanu, D., Jajarmi, A., Bonyah, E., Hajipour, M.: New aspects of the poor nutrition in the life cycle within the fractional calculus. Adv. Differ. Equ. (2018). https://doi.org/10.1186/s13662-018-1684-x

28. Jajarmi, A., Baleanu, D.: Suboptimal control of fractional-order dynamic systems with delay argument. J. Vib. Control 24(12), 2430-2446 (2018)

29. Khan, N.A., Razzaq, O.A., Hameed, T., Ayaz, M.: Numerical scheme for global optimization of fractional optimal control problem with boundary conditions. Int. J. Innov. Comput. Inf. Control 13(5), 1669-1679 (2017)

30. Goulart, A.G.O., Lazo, M.J., Suarez, J.M.S., Moreira, D.M.: Fractional derivative models for atmospheric dispersion of pollutants. Phys. A, Stat. Mech. Appl. 477, 9-19 (2017)

31. Salahshour, S., Allahviranloo, T., Abbasbandy, S.: Solving fuzzy fractional differential equations by fuzzy Laplace transforms. Commun. Nonlinear Sci. Numer. Simul. 17, 1372-1381 (2012)

32. Mondal, S.P., Roy, T.K.: First order linear homogeneous fuzzy ordinary differential equation based on Lagrange multiplier method. Int. J. Soft Comput. Appl. 2013, 1-17 (2013). https://doi.org/10.5899/2013/jsca-00032

33. Khan, N.A., Riaz, F., Razzaq, O.A.: An operator method for finding the solution of linear fractional order fuzzy differential equations. Prog. Fract. Differ. Appl. 2(1), 41-54 (2016)

34. Ren, J., Sun, Z.Z., Dai, W.: New approximations for solving the Caputo-type fractional partial differential equations Appl. Math. Model. 40(4), 2625-2636 (2016)

35. Bishehniasar, M., Salahshour, S., Ahmadian, A., Ismail, F., Baleanu, D.: An accurate approximate-analytical technique for solving time-fractional partial differential equations. Complexity 2017, Article ID 8718209 (2017). https://doi.org/10.1155/2017/8718209

36. Anagnost, J.J., Desoer, C.A.: An elementary proof of the Routh-Hurwitz stability criterion. Circuits Syst. Signal Process. 10(1), 101-114 (1991)

37. Gopal, M.: Control Systems: Principles and Design, 2nd edn. Tata McGraw-Hill, New Delhi (2002)

38. Shang, D., Guo, X.: Adams predictor-corrector systems for solving fuzzy differential equations. Math. Probl. Eng. 2013, Article ID 312328 (2013). https://doi.org/10.1155/2013/312328

39. Ahmad, M., Hasan, M.K., Abbasbandy, S.: Solving fuzzy fractional differential equations using Zadeh's extension principle. Sci. World J. 2013, Article ID 454969 (2013). https://doi.org/10.1155/2013/454969

40. Salahshour, S., Allahviranloo, T., Abbasbandy, S., Baleanu, D.: Existence and uniqueness results for fractional differential equations with uncertainty. Adv. Differ. Equ. 2012, 112 (2012). https://doi.org/10.1186/1687-1847-2012-112

\section{Submit your manuscript to a SpringerOpen ${ }^{\circ}$ journal and benefit from:}

- Convenient online submission

- Rigorous peer review

- Open access: articles freely available online

- High visibility within the field

- Retaining the copyright to your article

Submit your next manuscript at $\gg$ springeropen.com 